Data Assimilation

\title{
Estimating optimal localization for sampled background error covariances of hydrometeor variables
}

\section{Mayeul Destouches $^{1}$ I Thibaut Montmerle ${ }^{2}$ \\ Yann Michel $^{3}$ । Benjamin Ménétrier ${ }^{4}$}

1,2,3 CNRM, Université de Toulouse, Météo-France, CNRS, Toulouse, France

${ }^{4}$ IRIT, Toulouse, France

\section{Correspondence}

Mayeul Destouches, Météo-France, CNRM/GMAP, 42 Avenue Coriolis, 31057

Toulouse Cedex, France

Email: mayeul.destouches@umr-cnrm.fr

\section{Present address}

Météo-France, CNRM/GMAP, 42 Avenue

Coriolis, 31057 Toulouse Cedex, France

\section{Funding information}

Ministère de l'Enseignement Supérieur, de la

Recherche et de l'Innovation, AMX grant;

INSU, LEFE HYDRE

\begin{abstract}
This is the peer reviewed version of the following article: Destouches, M, Montmerle, T, Michel, Y, Ménétrier, B. Estimating optimal localization for sampled background-error covariances of hydrometeor variables. QJR Meteorol Soc. 2021; 147: 74-93., which has been published in final form at https://doi.org/10.1002/qj.3906. This article may be used for non-commercial purposes in accordance with Wiley Terms and Conditions for Use of Self-Archived Versions. This article may not be enhanced, enriched or otherwise transformed into a derivative work, without express permission from Wiley or by statutory rights under applicable legislation. Copyright notices must not be removed, obscured or modified. The article must be linked to Wiley's version of record on Wiley Online Library and any embedding, framing or otherwise making available the article or pages thereof by third parties from platforms, services and websites other than Wiley Online Library must be prohibited.
\end{abstract}




\section{ABSTRACT}

Kilometric scale Numerical Weather Prediction addresses the challenge of forecasting accurately clouds and precipitations. Ensemble-based data assimilation methods make use of background error covariances that are sampled from an ensemble of forecasts. These methods can be considered in order to include hydrometeor variables and their flow-dependent error covariances in the data assimilation system. Yet, because of limited ensemble size, rank deficiency of the resulting covariances and sampling noise occur, which can be mitigated by a localization procedure. In order to optimally localize covariances for hydrometeor variables, a previous work by the authors has been extended. This approach estimates localization as a linear filtering on covariances, optimal in the sense of minimizing sampling noise. The zero-variance and the high spatial variability issues met with hydrometeor variables are addressed by using an improved method for spatial sampling, based on geographical masks. Diagnosed optimal horizontal localization lengths appear to be much shorter for hydrometeors than for other classical thermodynamic variables. Conversely, we report optimal vertical localization to be very broad for precipitating species. Great variability between different meteorological situations has also been noticed, which reflects the high flow dependency of hydrometeor forecast errors. This suggests that ensemble-based data assimilation schemes that consider hydrometeors as control variables shall adopt more refined localization schemes than the common "one-size-fits-all" approach.

Keywords - Hydrometeor, background error covariances, optimal localization, ensemble data assimilation

\section{1 | INTRODUCTION}

Cloud cover and rainfall forecasting benefits from the development of convective-scale numerical weather prediction models with dedicated microphysics schemes, which describe the time evolution of three-dimensional hydrometeor contents. As other prognostic variables, hydrometeors require an appropriate initialization.

Optimal initial conditions are usually determined through the data assimilation (DA) process. Operationally, most convective-scale models include dynamic and thermodynamic variables such as horizontal winds, surface pressure, temperature and specific humidity (hereafter conventional variables) in the control variable of the DA process (Gustafsson et al., 2018). Hydrometeors, on the other hand, are usually not analyzed and simply kept unchanged through the DA process. This difference of treatment can lead to physical imbalances in the initial state, and spurious microphysical adjustments in the first time steps of the model integration.

Recently, a lot of research focused on the benefit of adding hydrometeor fields in the control variable of different DA systems. Snyder and Zhang (2003) used a rainwater control variable to assimilate simulated radar data, followed by Dowell et al. (2004) with real radar observations. Zhang et al. (2013) added five hydrometeor species in the control variable of the Maximum Likelihood 
Ensemble Filter to assimilate precipitation-affected satellite data. A positive impact on the prediction skill was observed, especially for rainfall location and intensity. Meng et al. (2019) showed a similar improvement in forecasting skill for precipitation when adding four hydrometeor variables in an hybrid ensemble-variational (EnVar) DA system.

Despite these results, the initialisation of hydrometeor fields remains a difficult task, both in theory and in practice (Bannister et al., 2019). Hydrometeor assimilation involves complex and highly nonlinear processes, in microphysics schemes and in observation operators (Errico et al., 2007; Auligné et al., 2011). Besides, hydrometeor mixing ratios should always remain positive. Non-Gaussian statistics naturally arise from these non-linearities and boundedness. Yet, almost all attempts to include hydrometeors in the control variable are based on Gaussian and near-linear frameworks. This article is no exception to the rule. The perspective of this work is to optimize the usual Gaussian-based DA methods to better handle hydrometeors, without fundamentally changing the algorithms.

In this context, unbiased forecast errors are fully described by their second-order moments, i.e. background error covariance matrices. The role of forecast errors, in particular their representation in the background error covariances, is prominent in DA (Bannister, 2008). As a matter of fact, an adequate description of forecast errors is required to properly weigh observations against the background state and to propagate the information spatially and among physical variables.

Hydrometeor forecast errors are especially flow dependent, due to the intrinsically heterogeneous and variable nature of hydrometeor fields (see for instance Michel et al., 2011). Part of this flow dependency can be described using a Monte-Carlo approach applied on an ensemble of forecasts, in order to sample the forecast error distribution at each run time. Time- and space-dependent background error covariances are then directly deduced from the ensemble. For hydrometeor assimilation, studies based on EnVar and ensemble Kalman filters (EnKFs) methods proved to outperform variational methods with static background error covariances (Johnson et al., 2015; Kong et al., 2018) and cloud analysis methods (Duda et al., 2019), thanks to the flow dependent retrieved increments. However, no operational applications have been reported yet.

\section{1 | Localization of background error covariances}

The computational cost of ensemble methods for systems with large state vectors limits the number of members in the ensemble. This limitation results in sampling noise, evidenced by spurious long-distance correlations in the estimated covariances. Filtering techniques are commonly used to remove these spurious correlations. The focus here is on the localization technique (Houtekamer and Mitchell, 2001; Hamill et al., 2001), which is used in both EnKF or EnVar systems. In practice though, localization is studied here for error auto-covariances in model space only, to be used e.g. in EnVar systems. In particular, domain localization is left out, as well as localization of covariances in observation space or in the cross-product of observation space and model space (as is done in many EnKF systems, see for instance Houtekamer and Zhang, 2016).

The main idea of localization in model space is to multiply the background error covariances by a localization factor, generally depending on the horizontal and vertical distances between the 
considered grid points (hereafter horizontal and vertical separations). The localization factor generally decreases from one at zero separation to zero for large separations. The signal is thus attenuated where the covariances and the signal-to-noise ratio are expected to be low. Note that in some cases, the localization factor does not depend solely on horizontal and vertical separations, for example when applying localization to covariances between different variables, or between different time steps.

A variety of methods have been proposed to diagnose optimal localization functions. Some can determine the whole shape of the function (e.g. Anderson, 2012; Flowerdew, 2015; Ménétrier et al., 2015a). Other methods rely on Gaussian-like localization functions and focus only on the optimal width of the Gaussian, the optimal localization length (or radius) (Sobash and Stensrud, 2013; Perianez et al., 2014; Kong et al., 2018). In most of these methods, localization is optimized for a specific context, be it the choice of an assimilation method (EnKF or EnVar flavours) or even the design of a full Observation System Simulation Experiments (OSSEs). The latter are used for instance by Sobash and Stensrud (2013), Lei et al. (2015), and Kong et al. (2018) with EnKF systems, and by Lorenc (2017) with a 3DEnVar system.

A different approach has been proposed by Ménétrier et al., 2015a (hereafter M15). The authors of this article propose a purely statistical method, based on the sampled ensemble only and independent from any other information about the system configuration. This independence from any operational system is interesting, as it allows to draw general conclusions that are hopefully not too much affected by a particular observational configuration or DA system. The methodology of M15 has been employed by several studies, with applications to operational weather models (Michel et al., 2016; Montmerle et al., 2018; Caron et al., 2018). This method is chosen here for optimal localization diagnosis.

\section{2 | Hydrometeor localization}

For hydrometeor background error covariances, shorter horizontal localization lengths are usually reported (compared to conventional background error covariances). Sobash and Stensrud (2013) for instance confirmed this result for microphysical variables through an OSSE experiment in an EnKF setting. Most attempts to include hydrometeor in the DA control variables follow the same paradigm, with horizontal localization radii of the order of $10 \mathrm{~km}$ in EnKF systems (Aksoy et al., 2009; Dowell et al., 2011; Sobash and Stensrud, 2013; Johnson et al., 2015). At least two reasons can justify the choice of shorter localization lengths for hydrometeor variables. Firstly, especially in EnKF systems, the localization radii are related to observations, rather than microphysical variables only. Tighter localization functions are thus empirically imposed to adapt to dense radar observation networks. Another reason for shorter localization length, can be found in the high spatial variability of hydrometeor background error covariances. Michel et al. (2011) for instance have studied the characteristics of multivariate dynamical and hydrometeor background error covariances. Inter alia, they showed on two meteorological situations that hydrometeor horizontal correlation length-scales were shorter than other variables. As localization length scales with correlation length (e.g. Flowerdew, 2015; Bannister et al., 2019), shorter optimal localization lengths should be deduced for hydrometeors.

So far, to the best of our knowledge, no objective localization diagnosis such as the method of 
M15 has been applied to hydrometeor fields in convective-scale models. Does this method confirm the choice of shorter localization lengths for hydrometeor covariances, compared to conventional ones? If so, is this result consistent over all microphysical variables, weather types and vertical levels? What prescriptions can be deduced for localization schemes involving hydrometeor control variables? The present study addresses these questions. In section 2, the theory of optimal localization from M15 is briefly recalled. The requirements for its extension to hydrometeors are detailed in section 3 . The experimental set-up is then presented in section 4 . Results are detailed in section 5 which ends with consequences for localization schemes involving hydrometeor forecast errors. The study is concluded in section 6.

\section{2 | THE OPTIMAL LOCALIZATION METHOD: THEORY AND PRACTICE}

\section{1 | Theory}

Let $x_{1}, \ldots, x_{N}$ be an ensemble of $N$ mutually independent forecasts of size $n$, drawn from the same random process (with $N$ greater than 4 ). The unbiased sampled covariance matrix $\widetilde{\mathbf{B}}$ of the ensemble is given by:

$$
\widetilde{\mathbf{B}}=\frac{1}{N-1} \sum_{k=1}^{N}\left(\mathbf{x}_{k}-\overline{\mathbf{x}}\right)\left(\mathbf{x}_{k}-\overline{\mathbf{x}}\right)^{T}
$$

where $\overline{\mathbf{x}}$ is the ensemble mean. A Schur (element-wise) product with a symmetric positive localization matrix $\mathbf{L}$ yields the filtered covariance matrix $\widehat{\mathbf{B}}$, commonly used in EnVar.

$$
\widehat{B}=L \circ \widetilde{B}
$$

where $\quad \widehat{B}_{i j}=L_{i j} \widetilde{B}_{i j}$ for all $i, j=1, \ldots, n$.

By defining the asymptotic matrix $\mathbf{B}^{\star}$ as the limit of $\widetilde{\mathbf{B}}$ when the ensemble size $N$ goes to infinity, one can define the optimal localization matrix as the $\mathbf{L}^{\text {opt }}$ matrix minimizing the distance $\mathbb{E}\left[\| \mathbf{L} \circ \widetilde{\mathbf{B}}-\mathbf{B}^{\star}||^{2}\right]$, where $\|\cdot\|$ denotes the Frobenius norm. Relying on results from linear filtering and centered moment estimation theories, the optimal localization matrix can be diagnosed from sampled estimates only (M15, their Equation 62):

$$
\begin{aligned}
L_{i j}^{\mathrm{opt}}= & \frac{(N-1)^{2}}{N(N-3)}-\frac{N}{(N-2)(N-3)} \frac{\mathbb{E}\left[\widetilde{\Xi}_{i j i j}\right]}{\mathbb{E}\left[\widetilde{B}_{i j}^{2}\right]} \\
& +\frac{N-1}{N(N-2)(N-3)} \frac{\mathbb{E}\left[\widetilde{B}_{i i} \widetilde{B}_{j j}\right]}{\mathbb{E}\left[\widetilde{B}_{i j}^{2}\right]}
\end{aligned}
$$


where $\cong$ is the sampled fourth-order centered moment of the ensemble and $1 \leq i, j \leq n$.

As outlined by Lorenc (2017), the diagnosed localizations are optimal in the sense of minimizing the distance between localized and asymptotic background error covariances. Hence, the method only addresses the problem of sampling noise, independently of the optimality of the resulting analysis. Optimal localization in terms of optimal analysis may differ from localization diagnosed here, depending on the relative weights given to observation and forecast errors, or on the quality of the ensemble (Lorenc, 2017).

This article focuses on optimal localization for auto-covariances of hydrometeors and of conventional variables. In particular, localization of inter-variable covariances is not studied there, though cross-covariances could also (at least theoretically) be studied with the M15 method.

\section{2 | Ergodicity assumptions}

\subsection{1 | Use of spatial averaging}

The expectation operators $\mathbb{E}[\cdot]$ in Equation 3 apply to both (independent) processes of asymptotic statistics generation and drawing $N$ members consistent with these asymptotic statistics (see M15 for details). Given that only one ensemble of $N$ members is available at a time, these expectations cannot be estimated directly. To approximate these operators, M15 formulate an ergodicity assumption by replacing the statistical expectations by horizontal and angular spatial averages. If the forecasts are discretized on a three-dimensional grid, all pairs $(i, j)$ of grid points of coordinates $\left(x_{i}, y_{i}, z_{i}\right)$ and $\left(x_{j}, y_{j}, z_{j}\right)$ can be partitioned into separation classes of given horizontal separation $d_{h}=\sqrt{\left(x_{i}-x_{j}\right)^{2}+\left(y_{i}-y_{j}\right)^{2}}$ and vertical model levels $z_{i}$ and $z_{j}$ (irrespectively of the order of $i$ or $j$, the problem being symmetric in $i$ and $j$ ). The horizontal separation distance $d_{h}$ is discretized to ensure there are enough pairs in each separation class. In order to keep computations affordable, a spatial sub-sampling approach is employed. A few representative pairs (at least 1000 in practice) are selected in each separation class, before computing the associated spatial average. Since the considered diagnostic package is conceived for possibly irregular grids, representative pairs are chosen by random, geographically homogeneous sampling in each class. The expectation operator is thus approximated as follows:

$$
\begin{aligned}
L^{\mathrm{opt}}\left(d_{h}, z_{i}, z_{j}\right)= & \frac{(N-1)^{2}}{N(N-3)}-\frac{N}{(N-2)(N-3)} \frac{\mu\left[\widetilde{\Xi}_{i j i j}\right]}{\mu\left[\widetilde{B}_{i j}^{2}\right]} \\
& +\frac{N-1}{N(N-2)(N-3)} \frac{\mu\left[\widetilde{B}_{i i} \widetilde{B}_{j j}\right]}{\mu\left[\widetilde{B}_{i j}^{2}\right]}
\end{aligned}
$$

where $\mu[\cdot]$ denotes an averaging operator on a random sub-sample from the separation class $\left(d_{h}, z_{i}, z_{j}\right)$, and $L^{\mathrm{opt}}\left(d_{h}, z_{i}, z_{j}\right)$ is now the diagnosed localization function defined on the three-dimensional discrete 
(a) Raw Localization

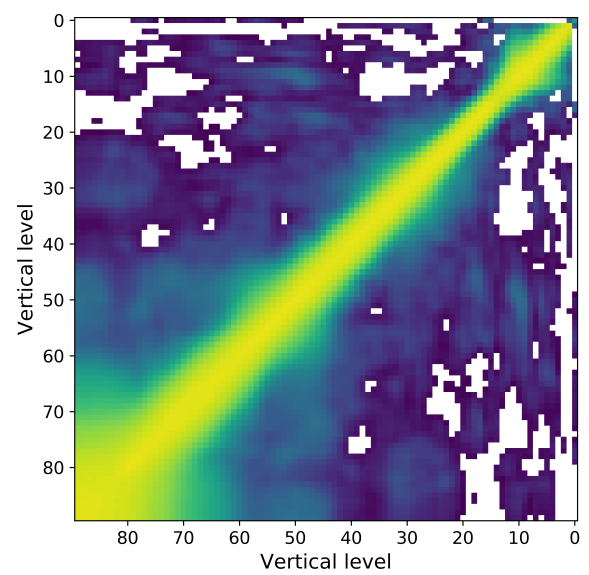

(b) Fit Analytical Localization

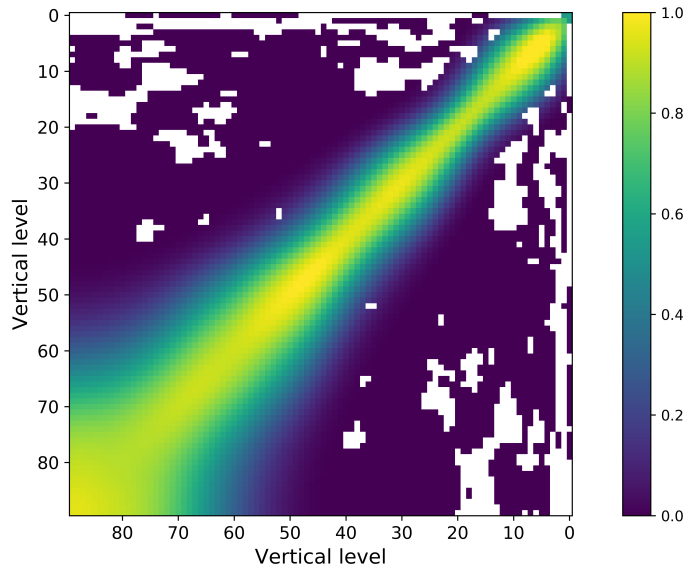

FIGURE 1 Raw (a) and fit analytical (b) localization functions for specific humidity. The localization is shown here when the horizontal separation $d_{h}$ is zero. It is diagnosed from an ensemble of 50 forecasts from the AROME EDA (cf. section 4), valid on November $15^{\text {th }}, 2018$ at 06 UTC. Missing values in white.

space of separation classes. For illustration purpose, figure 1(a) shows a diagnosed localization function in $\left(z_{i}, z_{j}\right)$ coordinates and for $d_{h}=0$, for specific humidity. Each color pixel represents an independent localization diagnosis. Equivalently, a different choice of coordinates to represent separation classes is given by vertical level $z=z_{i}$, vertical separation $d_{z}=z_{j}-z_{i}$ and horizontal separation $d_{h}$. Localization in figure $2(\mathrm{a})$ is shown in $\left(d_{h}, d_{z}\right)$ coordinates for instance.

\subsection{2 | Decomposition into ergodic sub-classes}

The ergodicity assumption may appear as a strong one to some readers. Actually, another interpretation of the spatial averaging can be given, allowing to relax the ergodicity assumption to the weaker assumption of ergodic decomposition.

Let us assume that a given separation class $C$ can be divided into $K$ ergodic sub-classes $C_{k}$, sampled with proportion $\alpha_{k}(1 \leq k \leq K)$, and that each subclass is correctly sampled. Then, the diagnosis $L^{\mathrm{opt}}\left(d_{h}, z_{i}, z_{j}\right)$ given by spatial averaging can be shown to verify:

$$
L^{\mathrm{opt}}\left(d_{h}, z_{i}, z_{j}\right) \approx \frac{\sum_{k=1}^{K} \alpha_{k} \mathbb{E}_{k}\left[B_{i j}^{\star 2}\right]}{\sum_{k=1}^{K} \alpha_{k} \mathbb{E}_{k}\left[\widetilde{B}_{i j}^{2}\right]}
$$


(a) Raw Correlation

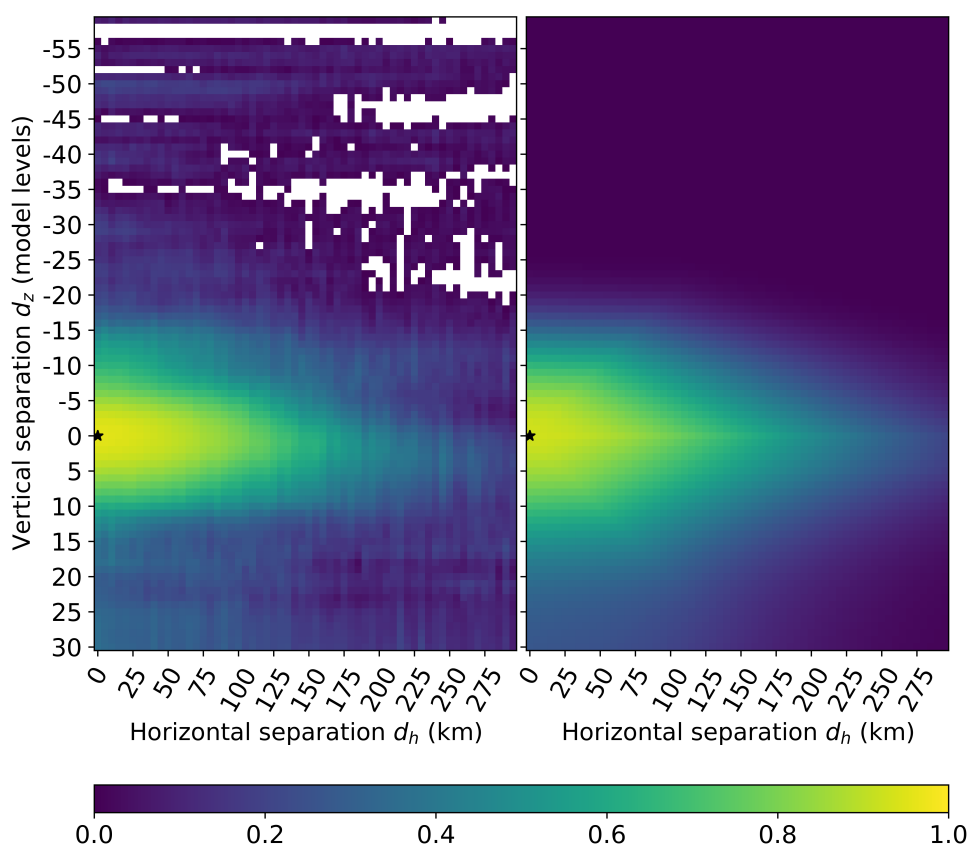

FIGURE 2 Same as figure 1, but localization is shown here in $\left(d_{h}, d_{z}\right)$ coordinates for model level $z=60$, that is around $430 \mathrm{hPa}$. The black star indicates the zero separation pair, where localization is close to 1 in both panels.

where $\mathbb{E}_{k}[\cdot]$ denotes expectation over the random process sampled by subclass $C_{k}$. The $\approx$ sign comes from imperfections in the estimations of expectations, due to limited size of each subclass. To better understand this formulation, Equations 30c and 57 of M15 can be combined to obtain the optimality criterion:

$$
\mathbb{E}\left[B_{i j}^{\star 2}\right]-L_{i j}^{\mathrm{opt}} \mathbb{E}\left[\widetilde{B}_{i j}^{2}\right]=0
$$

which implies that

$$
L_{i j}^{\mathrm{opt}}=\frac{\mathbb{E}\left[B_{i j}^{\star 2}\right]}{\mathbb{E}\left[\widetilde{B}_{i j}^{2}\right]} .
$$

Hence the right-hand side of Equation 5 is almost an average of sub-diagnoses $\mathbb{E}_{k}\left[B_{i j}^{\star 2}\right] / \mathbb{E}_{k}\left[\widetilde{B}_{i j}^{2}\right]$. 
Plus, it can be shown that this "average" actually minimizes

$$
\sum_{k=1}^{K} \alpha_{k} \mathbb{E}_{k}\left[\left(L_{i j} \widetilde{B}_{i j}-B_{i j}^{\star}\right)^{2}\right],
$$

which is the natural cost function to estimate a single optimal value of localization to be applied across different covariance statistics.

In practice, this interpretation of optimality is useful when diagnosing optimal localization over a domain where several weather situations co-exist. In this case, each sub-diagnosis correspond to each local weather situation, and the final diagnosis is the optimal combination of local diagnoses, following Equation 5.

\subsection{3 | Consistency tests}

Though these hypotheses of ergodicity are impossible to validate explicitly, a few a posteriori checks of consistency can be performed. These checks are consistent with both interpretations of the spatial averaging (sections 2.2.1 and 2.2.2). For the sake of clarity, we limit ourselves to the first interpretation (ergodicity assumption).

From sampled estimates $\mu\left[\widetilde{B}_{i j}^{2}\right], \mu\left[\widetilde{B}_{i i} \widetilde{B}_{j j}\right]$ and $\mu\left[\widetilde{\Xi}_{i j i j}\right]$, and under the assumption that $\mu[\cdot]=\mathbb{E}[\cdot]$, it is possible to retrieve the asymptotic quantities $\mathbb{E}\left[B_{i j}^{\star 2}\right]$ and $\mathbb{E}\left[B_{i i}^{\star} B_{j j}^{\star}\right]$.

The consistency tests consist in verifying a few basic statistical properties that should be true for these asymptotic statistics. Failures in passing these tests occur when the estimation $\mu[\cdot]=\mathbb{E}[\cdot]$ is too rough. Before issuing a diagnosis, we verify that:

$$
\begin{array}{r}
0 \leq \mathbb{E}\left[B_{i i}^{\star} B_{j j}^{\star}\right] \\
\mathbb{E}\left[B_{i j}^{\star}\right]^{2} \leq \mathbb{E}\left[B_{i j}^{\star 2}\right] \\
\mathbb{E}\left[B_{i j}^{\star 2}\right] \leq \mathbb{E}\left[\widetilde{B}_{i j}^{2}\right]
\end{array}
$$

Equation 10 is Hölder's inequality and implies that $0 \leq \mathbb{E}\left[B_{i j}^{\star 2}\right]$. This guarantees that $L_{i j}^{\text {opt }} \geq 0$ (cf. Equation 7), but is more constraining than mere positivity of diagnosed localization. Similarly, Equation 11 comes from sampling theory and guarantees that $L_{i j}^{\text {opt }} \leq 1$.

Missing values of raw localization can be seen in upper model levels in figures 1(a) and 2(a). These missing values correspond to separation classes where one at least of these inequalities is not verified (Equations 9 or 10 for most classes). Fortunately, this phenomenon is generally observed in zones of small sample correlation, where optimal localization is expected to be low. Surrounding separation classes have weak values of localization, so that the global shape of the localization function is not affected by these rejections. 
In the experiments presented in this article, the use of these consistency tests, the spatial consistency of results across separation classes, and the robustness to changes in spatial sampling comforts the idea that the assumption of ergodicity (or ergodic decomposition) is reasonable.

\section{3 | Zero separation localization}

Equation 4 does not enforce the localization to be 1 at zero separation, contrary to what could be expected from common practices in the literature. Even in the case of a Gaussian distributed ensemble, the theoretical value of localization at zero separation is $(N-1) /(N+1)$ (Equation 68 in M15). As a result, the corresponding estimator of variance differs from the standard unbiased estimator. This is because the optimal value of $L_{i i}$ minimizes the mean squared error $\mathbb{E}\left[\left(L_{i i} \widetilde{B}_{i i}-B_{i i}^{\star}\right)^{2}\right]=$ $\mathbb{E}\left[L_{i i} \widetilde{B}_{i i}-B_{i i}^{\star}\right]^{2}+\operatorname{Var}\left(L_{i i} \widetilde{B}_{i i}-B_{i i}^{\star}\right)$ which includes a variance term in addition to the squared bias. The fact that optimal localization $L_{i i}$ is strictly lower than 1 means that accepting a certain bias can be more than outweighed by the associated reduction in variance.

Small values of localization (even below 0.5) at zero separation are thus possible. According to the second term in Equation 4, this behavior indicates high kurtosis in the ensemble. In practice, such high values of kurtosis only concern hydrometeor distributions, which exhibit a peak at 0 and large tails evidenced by a few extreme values. As detailed in section 2.4, this hydrometeor specificity does not prevent from diagnosing localization lengths for hydrometeors. Indeed, applying a localization function that is strictly less than 1 at zero separation amounts to first reducing the ensemble spread (according to localization at zero separation), and then applying a normalized localization function. While the optimal localization at zero separation may still be an interest for future studies, zero separation diagnoses are not discussed further in the rest of the paper. Indeed, the ensemble spread might be modified anyway by an inflation scheme later in the DA process, depending on the considered system. Hereafter, the focus is put on optimal localization lengths.

\section{4 | From localization functions to localization lengths}

We propose to reduce the information contained in diagnosed localization functions to a length-scale when convenient:

- In order to easily visualize the results and compare them across different variables, or different meteorological situations;

- In order to provide relevant information, simple enough to be compatible with predefined localization schemes in DA systems.

In sections 2.1 and 2.2, localization is diagnosed as a function of vertical level $z$, vertical separation $d_{z}$ and horizontal separation $d_{h}$. This section explains how horizontal and vertical localization length profiles, $r_{h}(z)$ and $r_{v}(z)$ respectively, can be extracted from the diagnosed localization function.

$r_{h}$ and $r_{v}$ profiles are chosen to minimize the distance between diagnosed localization (hereafter 
raw localization) and an analytical Gaussian-like localization described by $r_{h}$ and $r_{v}$. In order to fit precisely the raw localization function, the analytical localization function is not constrained to be 1 at zero separation. A normalization factor $\alpha(z)$ is introduced to adjust this zero separation value.

The Gaspari and Cohn correlation profile (from Gaspari and Cohn, 1999) is chosen for the analytical localization, both in vertical and horizontal directions. This function approximates a Gaussian correlation function by a piece-wise fifth-order polynomial function of compact support, and is commonly used for localization purposes (e.g. Buehner, 2005). Therein, localization lengths refer to the Daley length $L_{D}$ of functions (Daley, 1993).

It should be noted that preserving the symmetry property of the analytical localization function is not straightforward if the localization lengths $r_{h}, r_{v}$ and the normalization factor $\alpha$ vary with model level $z$. A few details on the computation of the analytical localization function are given in appendix A. Besides, a penalty term for sharp profiles is added in the fit process, to avoid over-fitting the raw localization function. More details are given in appendix B. Note also that diagnosed localizations are generally not positive definite, which motivates their empirical fit by analytical functions. The fit step increases the robustness of the diagnoses by aggregating them, and is generally non-sensitive to large-separation missing values evoked in section 2.2.

Typical localization length profiles are shown in figure 3 . The corresponding analytical localization function is shown in figure 1 and 2 , side by side with raw localization. The analytical localization correctly reproduces the features observed in raw localization, at least for short separation distances. The symmetry of both raw and analytical localization functions is clearly visible in figure 1. 
(a)

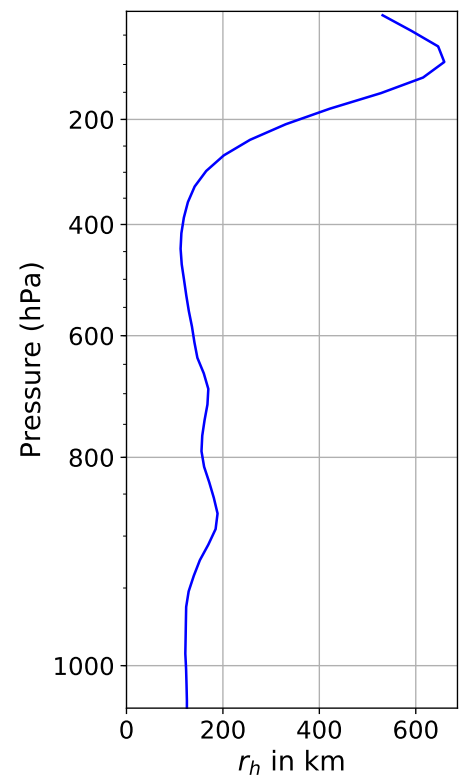

(b)

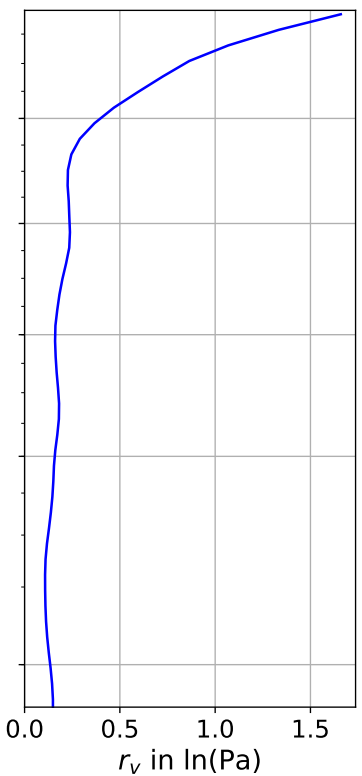

FIGURE 3 Diagnosed localization lengths as a function of vertical level, for specific humidity, same case as figure 1. (a) Horizontal and (b) vertical localization lengths.

\section{3 | EXTENSION TO HYDROMETEOR VARIABLES}

\section{1 | The issue of zero variances}

Applying the method of M15 to hydrometeor fields requires a few adaptations. By nature, hydrometeor fields are heterogeneous. As a matter of fact, hydrometeor mixing ratios are frequently zero where thermodynamic conditions below saturation prevail. When such a zero value is found at grid point $i$ in every ensemble member, the associated sampled variance $\widetilde{B}_{i i}$ is zero. Hence, sampled covariances $\widetilde{B}_{i j}$ are zero, and possibly the expectation of squared sampled covariances $\mathbb{E}\left[\widetilde{B}_{i j}^{2}\right]$ as well. Yet, Equation 3 requires that the expectation $\mathbb{E}\left[\widetilde{B}_{i j}^{2}\right]$ be non-zero, otherwise the fractions in the expression of optimal localization are undefined.

To better understand this limitation, one can refer to the optimality criterion of Equation 6 . When $\mathbb{E}\left[\widetilde{B}_{i j}^{2}\right]$ is zero, the value of the expression does not depend on $L_{i j}$. Since there is no covariance to be localized, $L_{i j}$ can indeed take any possible value without impacting the filtered covariances. This reasoning can be applied to the resulting analysis increments as well: if all ensemble members are equal on a given grid point, the increment at this grid point is necessarily zero (in a pure ensemble scheme), independently of the chosen localization. Consequently, there is no optimal localization $L_{i j}^{\text {opt }}$, 
and concerned pairs $(i, j)$ of grid points can just be excluded from the diagnosis.

\section{2 | Sampling hydrometeor fields more efficiently}

The discussion in previous section is essentially theoretical, since a statement such as $\mathbb{E}\left[\widetilde{B}_{i j}^{2}\right]=0$ cannot be evaluated in practice. The actual problem to be answered is what to do with separation classes where the expectations are estimated as zero: $\mathbb{E}\left[\widetilde{B}_{i j}^{2}\right] \approx \mu\left[\widetilde{B}_{i j}^{2}\right]=0$, where $\mu$ is the sampling-averaging operator over the separation class of pair $(i, j)$ (see Equation 4). A cautious choice is to discard such separation classes from the diagnosis. This questions in turn the validity of the estimation: what if we just under-sampled the separation class? What if we missed some non-zero pairs in the separation class? Then, the separation class would have been wrongly rejected, while a diagnosis might have been provided. Even then, how much confidence could one have on a diagnosis based on just a few grid points? To avoid such problems, more robust estimations of the expectations are needed. This section shows how sampling directly non-zero variance pairs improves the robustness of the estimations.

Let $C=\left(d_{h}, z_{i}, z_{j}\right)$ be a separation class. Let $\mathcal{I} \subset C$ be a random subset of $C$. Then the expectation $\mathbb{E}\left[\widetilde{B}_{i j}^{2}\right]$ can be estimated by ergodicity:

$$
\mu\left[\widetilde{B}_{i j}^{2}\right]=\frac{1}{\operatorname{Card}(\mathcal{I})} \sum_{(i, j) \in \mathcal{I}} \widetilde{B}_{i j}^{2},
$$

where $\operatorname{Card}(\mathcal{I})$ designates the cardinal of set $\mathcal{I}$. In practice, $\mathcal{I}$ is sampled so that $\operatorname{Card}(\mathcal{I}) \ll \operatorname{Card}(C)$, in order to enable faster computations and to limit memory usage. The set $\mathcal{I}$ can be partitioned into pairs of zero variances and pairs of non-zero variances: $\mathcal{I}=\mathcal{I}_{0} \cup \mathcal{I}_{+}$with

$$
\begin{aligned}
& \mathcal{I}_{0}=\left\{i, j \in \mathcal{I} \mid \widetilde{B}_{i i}=0 \text { or } \widetilde{B}_{j j}=0\right\} \\
& \mathcal{I}_{+}=\mathcal{I} \backslash \mathcal{I}_{0}=\left\{i, j \in \mathcal{I} \mid \widetilde{B}_{i i}>0 \text { and } \widetilde{B}_{j j}>0\right\}
\end{aligned}
$$

And similarly $C=C_{0} \cup C_{+}$. This partition of $\mathcal{I}$ enables to simplify the fractions in Equation 4 . For its second term for instance:

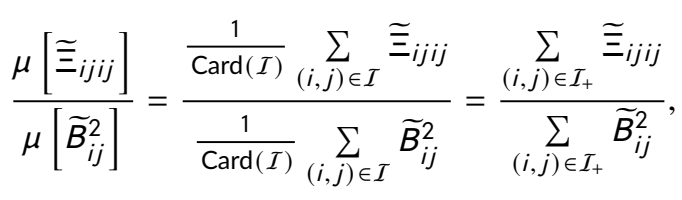

since zero variances imply zero fourth-order moments. A similar result is obtained for the third term of Equation 4. As a consequence, localization diagnosed from set $\mathcal{I}$ is the same as localization diagnosed from set $\mathcal{I}_{+}$. This argues in favour of rejecting zero-variance pairs (in $\mathcal{I}_{0}$ ) from the sampling step. By 
sampling pairs directly in $C_{+}$while keeping the same number of sampled pairs, the effective sample size is increased by a factor $\operatorname{Card}(C) / \operatorname{Card}\left(C_{+}\right)$on average, which can be quite important if only a small part of the geographical domain has a non-zero hydrometeor content. The diagnosis is thus made more robust, by sampling more densely the separation class. Note however that the increase in effective sample size obtained by sampling more densely is partly mitigated by higher spatial correlations among the sampled pair. The given increase factor $\operatorname{Card}(C) / \operatorname{Card}\left(C_{+}\right)$may thus be an upper bound.

\section{3 | Excluding zero variances in practice}

A rather straightforward way of sampling only non-zero variance pairs consists in excluding zero variance points individually, before forming pairs. Yet, due to rounding errors in the different computations, a significant part of variances may not be exactly zero, but still be exceedingly small and non physically significant. A threshold has to be chosen, below which standard deviation are considered to be zero, leading to rejection of the grid points. A threshold value of $10^{-12} \mathrm{~kg} \mathrm{~kg}^{-1}$ in ensemble standard deviation is chosen. Several arguments are given below to support this choice.

1. A $10^{-12}$ standard deviation corresponds to a variance of $10^{-24}$, which is below the accuracy of double precision computations.

2. Figure 4 shows that the accepted grid points gather around high concentration zones, which should not be the case for numerical noise.

3. This choice is confirmed by looking at the implied geographical three-dimensional masks on variances. As shown in figure 5, the chosen threshold is located in a range of values (from $10^{-11}$ to $10^{-17}$ ) where the mask is almost non sensitive to the choice of threshold .

4. This threshold is relevant across all hydrometeor types, all levels and all considered weather situations. 


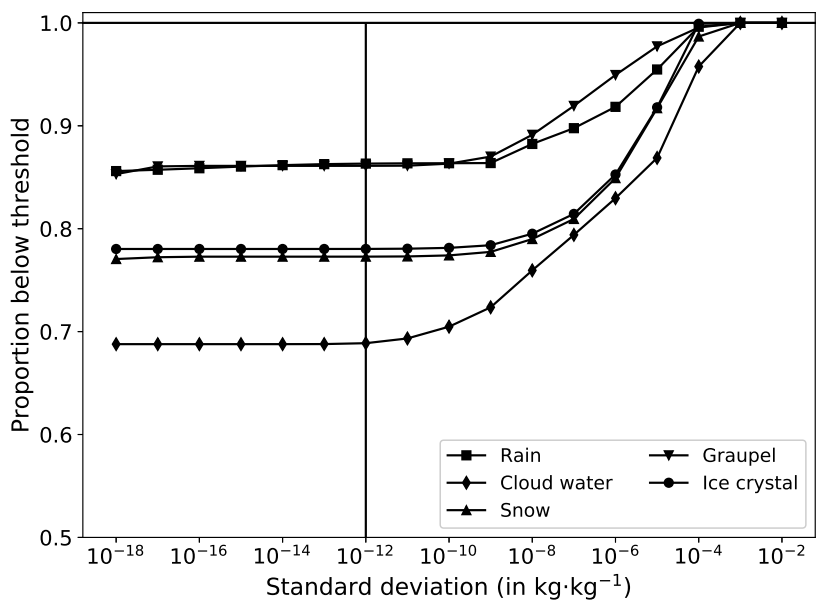

FIGURE 5 Empirical cumulative distribution function of hydrometeor sampled variances in ensemble for case 7 and across all vertical levels. The horizontal scale is logarithmic.

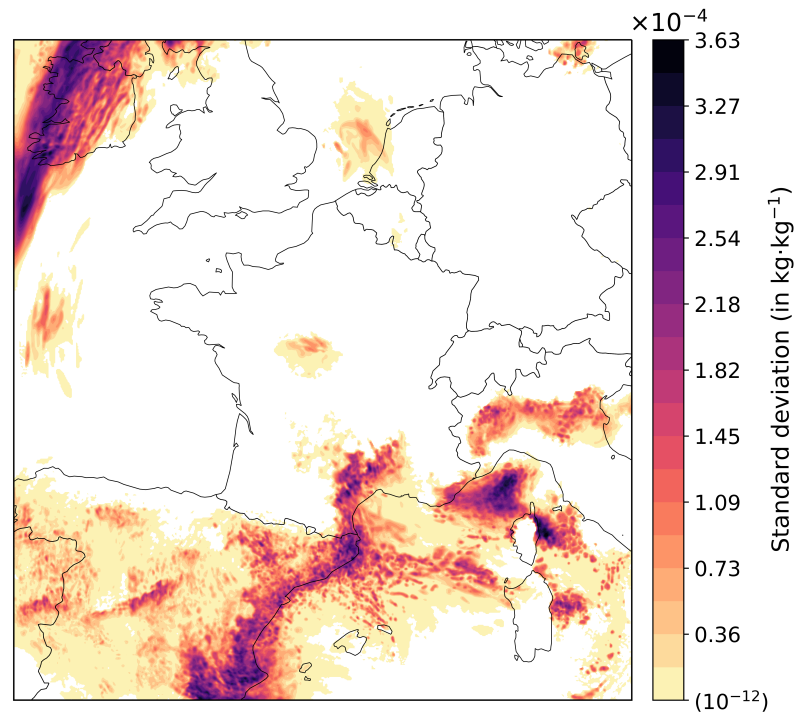

FIGURE 4 Standard deviations of cloud water $\left(q_{l}\right)$ content at model level 62 (around $850 \mathrm{hPa}$ ) over the AROME domain. The standard deviations are sampled from a 50 member ensemble from the AROME EDA system, valid at 6 UTC in November $15^{\text {th }}, 2018$. Standard deviations are masked below the threshold value $10^{-12} \mathrm{~kg} \mathrm{~kg}^{-1}$. 


\subsection{Acceptance tests for localization lengths}

Once the diagnosis is performed, a test of acceptance is carried out level by level on $r_{h}$ and $r_{v}$ profiles. Indeed, the fit of the analytical function is performed globally, so that $r_{h}(z)$ and $r_{v}(z)$ are defined across all levels. The acceptance test aims at rejecting levels where the raw localization was not correctly fitted by the analytical localization function. This typically happens when the shape of the raw localization function strongly differs from a Gaussian, when the raw localization diagnosis is very noisy, with no sign of decay with increasing separation distance, or when the diagnosis could simply not be performed because of absence of hydrometeor. The former cases are evidenced by unusually high residuals of the fit. The latter is evidenced when too few localization diagnoses are available to describe the raw localization function at a given level. More details on the acceptance tests are given in appendix C.

Even though this quality control step only affects hydrometeor variables in practice, the whole localization diagnosis is performed identically for hydrometeor variables and conventional variables.

\section{4 | EXPERIMENTAL SET-UP}

\section{1 | The AROME EDA}

Given that localization diagnoses only require information from an ensemble of forecasts, ensemble generation deserves a specific attention. All ensembles used in this study are generated by the AROME Ensemble Data Assimilation (EDA) system, which evolves 50 members through 3 hour cycles of DA and forecast steps. Such a system aims to simulate the error evolution of the AROME-France DA system (Seity et al., 2011) through the addition and propagation of both observation and model perturbations during the DA cycling. In addition to what is classically done at global scale (e.g Berre and Desroziers, 2010), perturbed lateral boundary conditions provided by the operational EDA at global scale based on the ARPEGE model are also used (Berre et al., 2015). No inflation is applied to the ensemble, but the method of Stochastically Perturbed Parametrization Tendencies (SPPT) is used to simulate model error (Palmer et al., 2009; Bouttier et al., 2012) with a horizontal length-scale of $500 \mathrm{~km}$. The water species are evolved by the single-moment microphysics scheme ICE3 (Lascaux $e t$ al., 2006). Besides mass mixing ratio of water vapour $q_{v}$, the ICE3 scheme describes the evolution of five hydrometeor variables: cloud water $q_{l}$, ice crystals $q_{i}$, rain $q_{r}$, snow $q_{s}$ and graupel $q_{g}$ (that also includes hail as large graupel particles). Compared to the deterministic AROME-France model, which nowadays runs at $1.3 \mathrm{~km}$ horizontal resolution and performs hourly DA cycles (Brousseau et al., 2016), the same vertical discretization of 90 levels is used, whereas a lower horizontal resolution of $3.2 \mathrm{~km}$ has been preferred to lower computational cost.

As the AROME EDA was not yet in operation at Météo-France, three cycling experiments have been performed over February 2018, May to October 2018 and November 2018, encompassing eight meteorological situations of interest. The eight studied ensembles are extracted at least 2 days after the beginning of each EDA cycled experiment, to make sure the system has reached an equilibrium state with an appropriate dispersion. 
As displayed in Equation 3, the absolute value of the diagnosed localization length depends of the ensemble size. Yet, the ratio of the retrieved lengths between hydrometeors and conventional variables should be less sensitive to such size, which keeps the results relevant for application to different ensemble-based DA systems.

\section{2 | Meteorological situations}

\begin{tabular}{|c|c|l|}
\hline$\#$ & Validity date & Weather type \\
\hline 1 & $2018 / 02 / 1103 \mathrm{~h}$ & Weather disturbance \\
2 & $2018 / 02 / 1303 \mathrm{~h}$ & Little rain winter case \\
3 & $2018 / 05 / 2115 \mathrm{~h}$ & Barometric swamp thunderstorms \\
4 & $2018 / 05 / 2815 \mathrm{~h}$ & Locally intense thunderstorms \\
5 & $2018 / 08 / 0912 \mathrm{~h}$ & Thunderstorms. \\
6 & $2018 / 10 / 1503 \mathrm{~h}$ & High Precipitation Event in South-Eastern France \\
7 & $2018 / 11 / 1506 \mathrm{~h}$ & Fog over northern France \\
8 & $2018 / 11 / 1606 \mathrm{~h}$ & Fog over northern France \\
\hline
\end{tabular}

TA B LE 1 Studied meteorological situations. Hours are given in UTC.

Eight cases are studied, and listed in table 1. The meteorological situations were chosen to be representative of different weather patterns implying different hydrometeor species over the AROME domain in 2018. Cases have been selected both in winter (two in February), in summer (one in August) and in mid-seasons (two in May, one in October, two in November). Half of the cases are mostly convective, for instance in the end of May, where intense thunderstorms developed over France. The August case has been chosen for being the most active in the month, in terms of number of detected lightning flashes. Finally, the October case is a High Precipitation Event in South France, with local rainfall reaching about $260 \mathrm{~mm}$ in $12 \mathrm{~h}$, which caused 14 casualties in the Aude department from the resulting flooding. More stratiform cloud cases were selected in November, with two situations of wide-spread fog over the North of France. The two winter cases are more common, with the passage of a weather disturbance with scattered showers over France, and a more dry but cloudy typical mid-latitude winter atmosphere.

\section{3 | Implementation of the localization diagnoses}

The localization diagnoses have been performed using the BUMP package developped by Benjamin Ménétrier (see acknowledgment section for details). A few technical details are given here.

As mentioned in section 2.2, separation classes are divided according to a discretized horizontal separation distance, inter alia. The horizontal resolution $\Delta d$ should be fine enough to properly inform on the decay of localization with horizontal separation. On the other hand, it should be consistent with the horizontal resolution of the ensemble $(3.2 \mathrm{~km})$, to make sure that there are enough pairs in a given 
separation class. Otherwise, estimation of expectations in Equation 4 might suffer from under-sampling. The horizontal class width is chosen as $\Delta d=5 \mathrm{~km}$ in these experiments, with horizontal separations ranging from $0 \mathrm{~km}$ to $300 \mathrm{~km}$.

Vertically, separation classes are naturally discretized by all possible pairs of levels.

For the sampling step, up to 10000 pairs are sampled and averaged for each separation class.

\section{4 | Confidence}

To have an idea of the robustness of the diagnoses, the whole process of localization profile estimation is performed ten times per variables, with different seeds of the random number generator. Changing the seed changes the random selection of geographical pairs $(i, j)$ that are used in each separation class, and finally changes the localization diagnosis. The variability of the results gives a coarse idea of the uncertainty due to spatial sampling. These uncertainty ranges are shaded in figure 6 . If less than half of the 10 diagnoses pass the acceptance tests at a given vertical level (section 3.4 and appendix C), the remaining diagnosed localization lengths at this level are screened out, and the result is regarded as not robust enough to be considered. Otherwise, the median value of the level is kept as diagnosed localization length, to get rid of potential outliers.

\section{5 | RESULTS: LOCALIZATION DIAGNOSES}

\section{1 | Horizontal localization}

Diagnosed horizontal localization lengths $\left(r_{h}\right)$ for November $15^{\text {th }}$ (first fog case) are presented in figure 6 . At first, $r_{h}$ uncertainty ranges look wider for hydrometeors: around $30 \%$, against $10 \%$ for conventional variables. Yet, the absolute width of the uncertainty range is similar, around $15 \mathrm{~km}$ for every variable and for most levels. The uncertainty range is omitted for the sake of clarity in figure 7 , where $r_{h}$ profiles from all meteorological cases are gathered for a selection of variables.

\subsection{1 | Dependence to vertical level}

Diagnosed horizontal localization lengths $r_{h}$ are strongly dependent on vertical level. This dependence is especially clear for hydrometeor variables, for which localization can be diagnosed for a fraction of vertical levels only. Different hydrometeor variables in the ensemble are gathered in different portions of the atmosphere: lower levels for cloud water and rain, upper levels for snow and ice crystals, all levels for graupels that gather a mix of melted particles essentially present in convective towers.

Where the diagnoses can be performed, $r_{h}$ values for hydrometeor remain level dependent. For $q_{l}$ for instance, in figure 6 , a peak is displayed around $850 \mathrm{hPa}$ with values greater than $200 \mathrm{~km}$, which is roughly 4 times the value below (around $1000 \mathrm{hPa}$ ) and above (around $750 \mathrm{hPa}$ ). We hypothesize that the diagnosed peak in $r_{h}$ is due to the passage of a linear cloud structure in the north-western part of the domain. This structure leaves its imprint on the map of sampled standard deviations in figure 4). This 
(a)

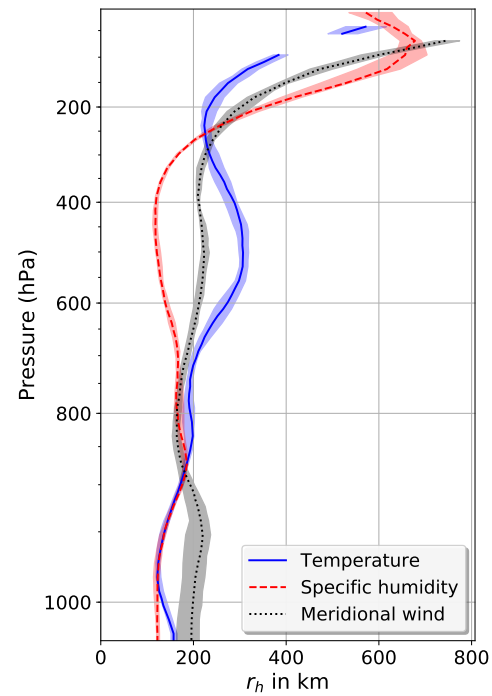

(b)

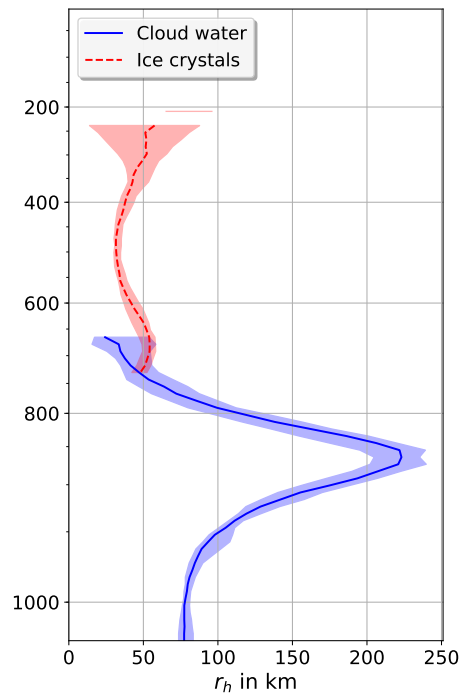

(c)

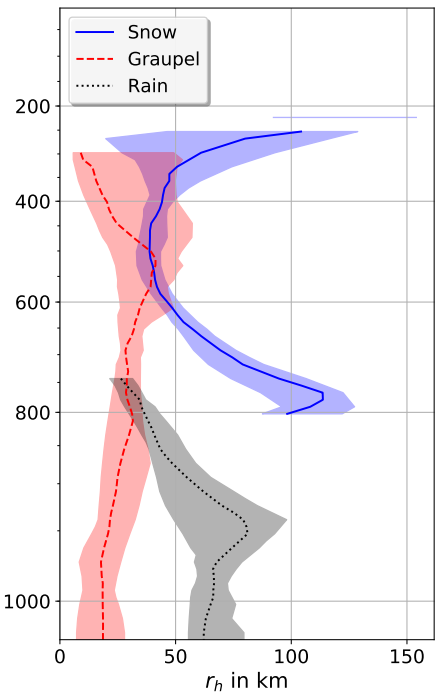

FIG URE 6 Diagnosed horizontal localization lengths diagnosed for November $15^{\text {th }}, 20186$ UTC case. Shaded areas show the interval between the $10^{\text {th }}$ and $90^{\text {th }}$ percentiles of a 10 run ensemble. Horizontal scales differ from one panel to another.

hypothesis is confirmed by performing separate diagnoses for the southern and northern halves of the domain. Only the northern diagnosis reproduces this peak in $r_{h}$ (not shown), associated with larger correlation lengths on average. So the diagnosis on the whole domain is the optimal combination of southern and northern diagnoses, as described in section 2.2.2.

Profiles of $r_{h}$ diagnosed for the other weather situations reveal similar vertical variability. Figure 7 shows that for each hydrometeor variable, at least one day exhibits a significantly vertical-dependent profile. This variability can be simply quantified for each profile by computing the ratio of maximum over minimum localization lengths (max over min ratio). This max over min ratio is almost always higher than 2, across all variables and times. Cloud water $r_{h}$ profiles in particular are often peaky, with a max over min ratio above 9.0 in 7 cases out of 8 . Other hydrometeor variables can be quite uniform, especially for rain, but frequently present a peak in localization at some altitude. This uniformity might be related to their precipitating nature, which implies similar error structures across vertical levels, and hence similar $r_{h}$.

For conventional variables, $r_{h}$ values rapidly increase from $100-300 \mathrm{~km}$ in the troposphere to $400-$ $700 \mathrm{~km}$ in the stratosphere. This phenomenon has already been noticed by Ménétrier et al. (2015b) and Montmerle et al. (2018). This could be partly a specific feature of the AROME-EDA, maybe related to coupling to a large scale model on the top of the atmosphere. It could also be related to the usual increase of correlation length scales with height (see for instance Caron and Buehner, 2018). 
(a) Temperature

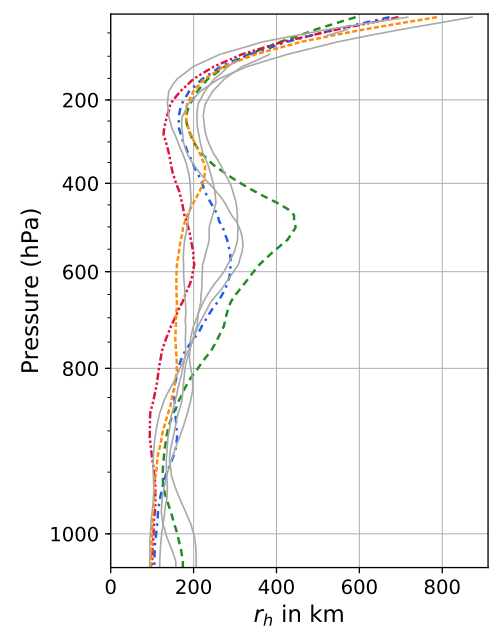

(d) Ice crystals

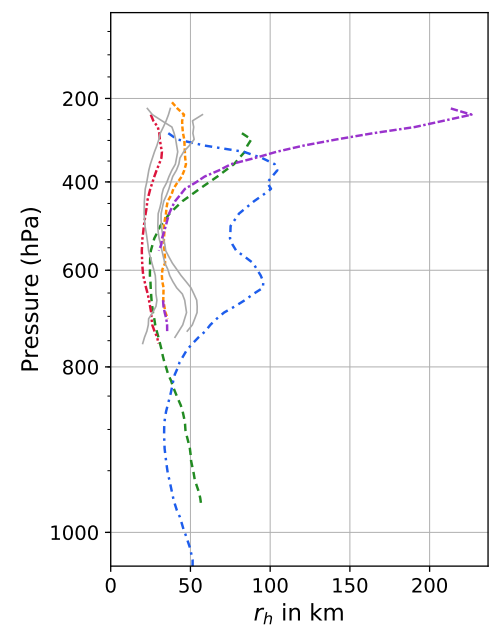

(b) Specific humidity

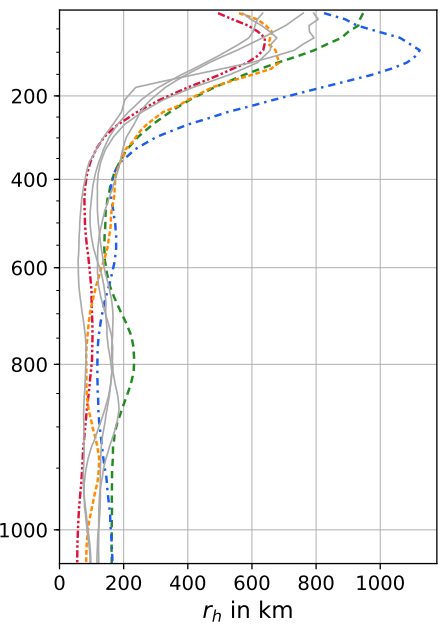

(e) Rain

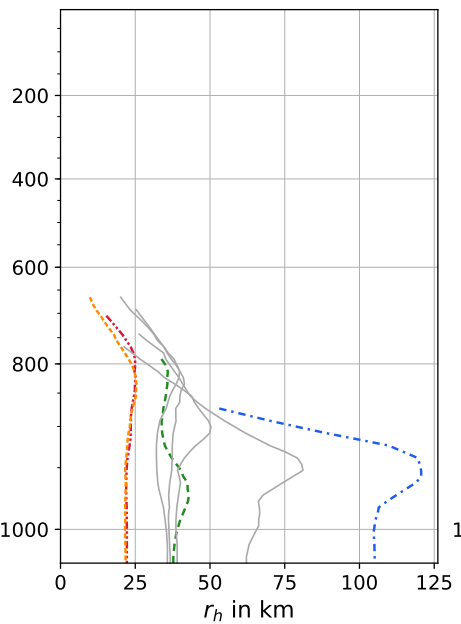

(c) Cloud water

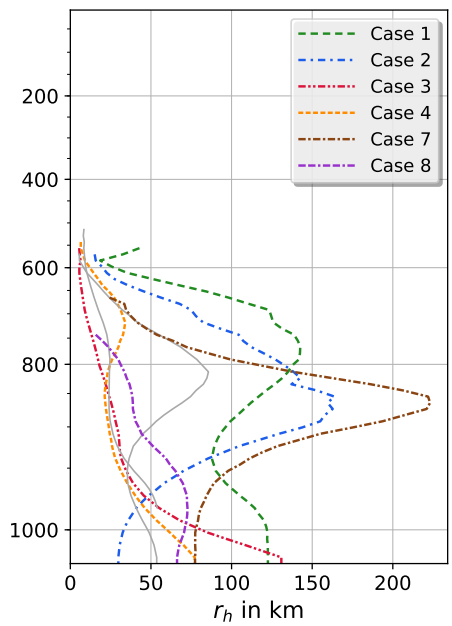

(f) Snow

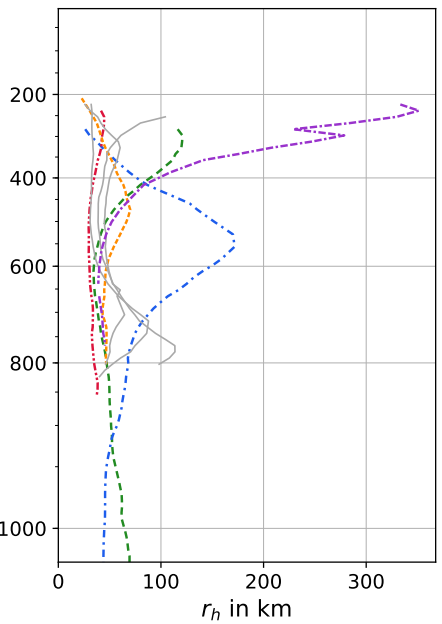

FIGURE 7 Diagnoses of horizontal localization lengths for 8 studied ensembles and 6 variables. Each profile is the median value from 10 runs. Data is not shown where more than half of the 10 runs fail to issue a diagnosis. Some days are enhanced by specific color and line style, see text for details. Horizontal scales differ between panels. 


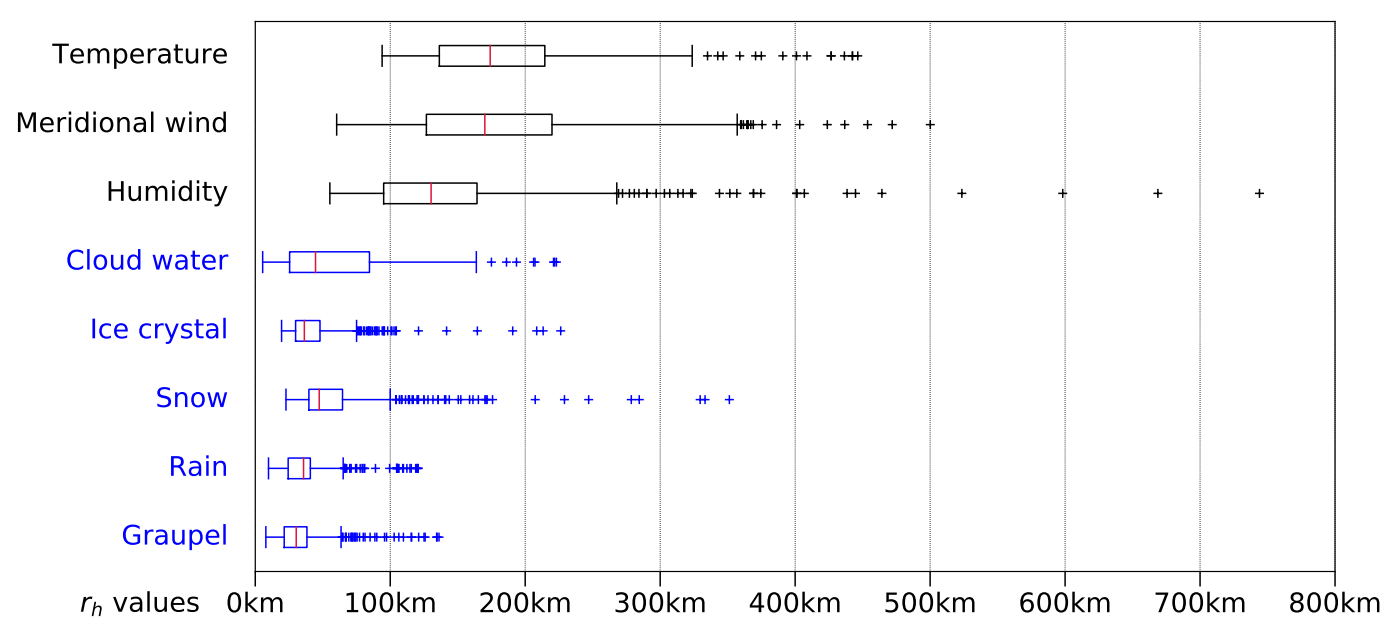

FIGURE 8 Range of values for $r_{h}$ for different variables, aggregated from all 8 studied days and vertical levels below $190 \mathrm{hPa}$. Localization lengths above $190 \mathrm{hPa}$ are discarded, since there is no hydrometeor diagnosis for comparison with conventional variables at these levels. The boxes extend from first quartile $Q 1$ to third quartile $Q 3$. The median value is shown in between. The whiskers extend on each side to the furthest points within $d \mathrm{~km}$ of box ends, where $d$ is 1.5 times the inter-quartile range $Q 3-Q 1$. Points farther away are marked with crosses.

\subsection{2 | Dependence to weather situation}

As shown in figure 7, the localization diagnosis depends on the considered meteorological situation. For instance, diagnosed $r_{h}$ are generally larger for winter cases (cases 1 and 2) than for the convective May cases (cases 3 and 4), for all considered variables. This difference is probably linked to similar differences in the correlation structures, more flattened in stratified winter situations. Hence, the variability of correlation structures with weather types, already noted by Brousseau et al. (2012) and Ménétrier et al. (2014) for instance, is reflected in the variability of the diagnosed localizations.

The daily variability of $r_{h}$ is comparable to, and maybe even greater than the seasonal variability. Two diagnoses can clearly differ from one day to the next, as shown by the two November profiles (fog cases 7 and 8) for cloud water in figure 7(c). The diagnosed profiles are similar in the first bottom layers of the atmosphere, where fog conditions are similar. However, the profiles rapidly diverge above the first few layers, due to the passage of the linear cloud structure previously mentioned in case 7 , which is absent of the domain in case 8 .

\subsection{3 | Range of values for horizontal localization}

Figure 8 summarizes the differences of diagnosed $r_{h}$ for all levels, cases and variables, by displaying on one figure the range of diagnosed values. Clearly, shorter $r_{h}$ (mainly $20 \mathrm{~km}$ to $90 \mathrm{~km}$ ) have been obtained for hydrometeors, compared to conventional variables (mostly $90 \mathrm{~km}$ to $220 \mathrm{~km}$, except in 


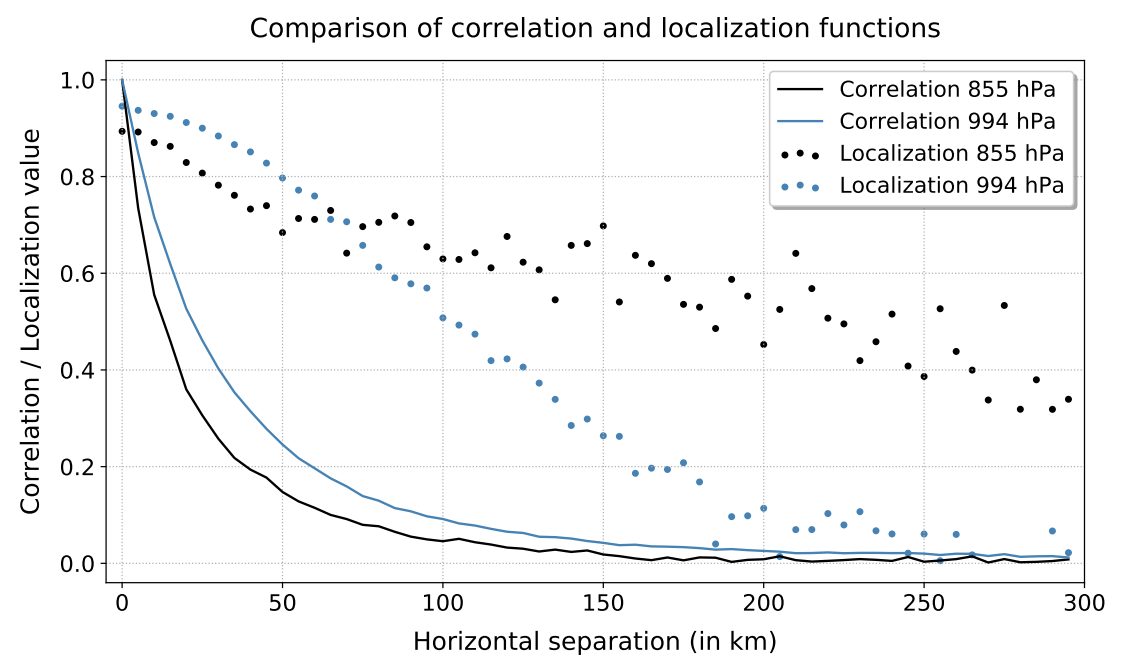

FIG URE 9 Horizontal section of mean sample correlation and optimal localization functions. The sections are extracted at two different altitudes and correspond to cloud water on figure 6 (b).

the stratosphere). The shortest values are consistently displayed for liquid rain and graupel, that are the condensate with the largest fall speed, whereas values for liquid cloud are more variable and can be larger. These results are quite consistent when compared day by day and level by level, with values for hydrometeors that are smaller compared to conventional variables by a factor 2 to 6 (not shown). Surface pressure diagnoses have been performed for comparison purposes and show a median value for $r_{h}$ around $940 \mathrm{~km}$, making surface pressure the conventional variable with largest horizontal localization length.

\subsection{4 | Relationship to correlation lengths}

The ambiguous relationship between correlation and localization is illustrated in figure 9, where sections of correlation and localization functions are displayed together. This figure evidences that broader mean sample correlation functions are not always associated to broader localization functions.

More generally, even though correlation structures can help to understand localization diagnoses, the vertical variability of $r_{h}$ cannot be fully explained by the underlying correlation functions. For instance, a peak of diagnosed $r_{h}$ is observed for cloud water in case 2 (February $13^{\text {th }}$ ), while no such feature can be observed in the sample correlation function around this altitude (not shown).

Similar results show that optimal vertical localization lengths cannot always be related to correlation length-scales (not shown). 
Case 7 - raw localization

(a) Temperature

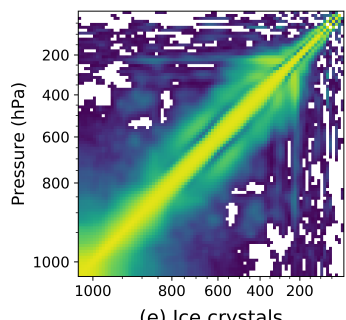

(e) Ice crystals

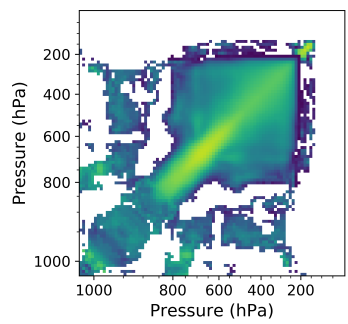

(b) Specific humidity

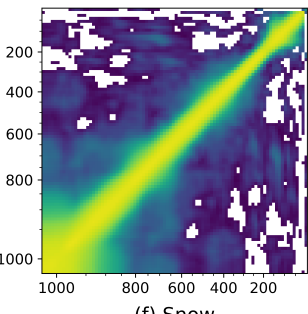

(f) Snow

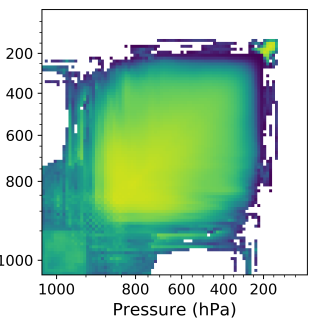

(c) Meridional wind

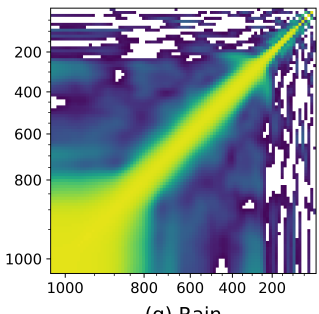

(g) Rain

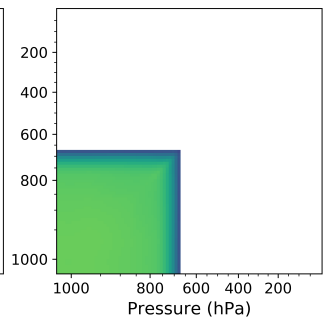

(d) Cloud water

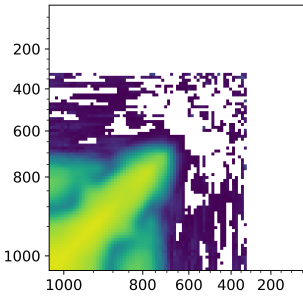

(h) Graupel

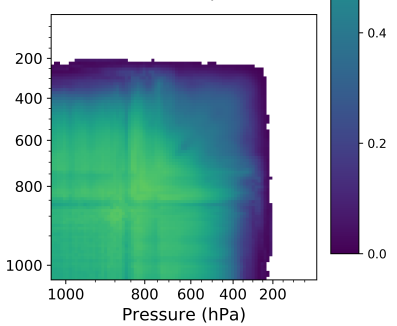

FIGURE 10 Matrices of raw localization at zero horizontal separation for case 7 (November $15^{\text {th }}$ ). The values shown here are the median of the 10 runs, where half at least of these runs give a non-missing value. This explains why (b) differs from figure 1(a).

\section{2 | Vertical localization}

\subsection{1 | Raw localization functions}

Figure 10 presents raw localization functions for conventional and hydrometeor variables, for case 7 (first November fog case).

A first interesting feature is the significantly lower localization $L_{i i}$ at zero separation (on the diagonals) for rain and graupel. Maximum value for these variables is around 0.77 , while it is $0.90-0.95$ for other hydrometeor variables, and around 0.96 (the value for Gaussian ensembles) for conventional variables. As mentioned in section 2.3, these rather low values may reflect heavy tails of graupel and rain distributions in the ensemble.

Another specificity of rain and graupel is the quasi uniform values in the vertical localization matrices. For both cases, raw localization decays only very slowly with increasing vertical separation, and drops suddenly to zero just before reaching the zone of non-definiteness (levels without hydrometeor content). The same phenomenon, to a lesser extent, is observed for snow. We suggest that these broad vertical localization structures are linked to the precipitating nature of these particular variables. This result is consistent with the large vertical correlation structures found for liquid rain by Michel et al. (2011).

These vertical uniformities of localization values are observed for all other cases as well, especially 

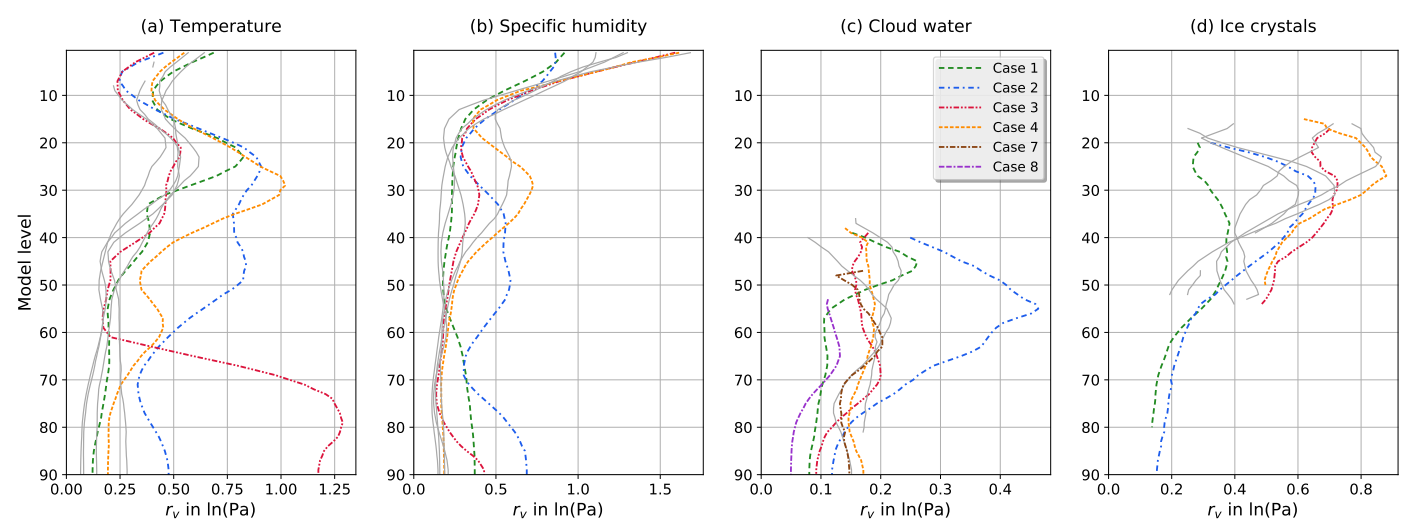

FIGURE 11 As figure 7 for vertical localization lengths.

for rain (not shown). Day-to-day variability mainly resides in the altitude of the transition level from high to zero localization values, which varies depending on the vertical stratification of the considered weather situation and on the considered hydrometeor.

\subsection{2 | Localization lengths}

Diagnosed profiles of $r_{v}$ are shown in figure 11 for all studied cases. No estimation is performed for precipitating variables, since their raw localization shapes differ too strongly from the analytical Gaussian shape.

Similarly to $r_{h}, r_{v}$ values depend on the vertical level, on the variable and on the weather situation. Most profiles present one or two peaks, in all displayed variables. For $q_{i}$ especially, one peak of maximum values is always observed between $550 \mathrm{hPa}$ and $300 \mathrm{hPa}$. These peaks correspond to the levels of maximum $q_{i}$ content. The two winter profiles for ice crystal almost reach surface level, while no diagnosis can be performed below $800 \mathrm{hPa}$ for summer and mid-season cases.

Contrary to $r_{h}$, no clear connection can be established between $r_{v}$ and convective activity. Case 2 (February $13^{\text {th }}$ ) profile exhibits especially large values for temperature, $q_{l}$, and to a lesser extent specific humidity. Yet, values do not seem to be especially larger for May convective cases, nor shorter for November stratified cases.

Another important difference with $r_{h}$ is the similarity of profiles for conventional variables and cloud variables $\left(q_{i}\right.$ and $q_{l}$ ), at least in terms of range of values. No significant difference can be shown here from one variable to another. From the surface to $600 \mathrm{hPa}, r_{v}$ values for $q_{i}$ and $q_{l}$ are gathered just below 0.2 (in $\ln (\mathrm{Pa})$ in this section), with extreme values above 0.4, consistently with $q_{v}$ values. Above $600 \mathrm{hPa}$, vertical lengths for $q_{i}$ extend from 0.2 to 0.8 , rather like temperature lengths. 


\section{3 | Consequences for localization schemes}

Here follows a list of possible localization schemes accounting for the results of this study, from the simplest to the most elaborate. All of them apply to convective-scale models that consider hydrometeor in their control variables.

1. Single localization scheme. This is one of the cheapest possible localization schemes in terms of computational cost: one value of $r_{h}$ and $r_{v}$, common for all variables, all levels, all days.

2. Variable-dependent localization scheme. The idea of such a scheme is not new, and has recently been advocated by Necker et al. (2020) for instance. This scheme is more expensive in terms of computer power, but the cost can be limited if some variables have a common localization. Typically, control variables could be divided into three sets: conventional variables, cloud variables and precipitating variables. The latter two (hydrometeors variables) should have shorter $r_{h}$ compared to conventional variables, by a factor 2 to 6 . On the other hand, precipitating hydrometeors could be only slightly localized in the vertical, with a large $r_{v}$ compared to other hydrometeors and conventional variables.

3. Variable-, level- and day-dependent localization scheme. Such an increase in complexity is required to significantly refine the localization scheme. This implies that an estimation of optimal localization should be performed every day, ideally before every DA cycle.

4. Flow-dependent localization. If the geographical domain of the convective-scale model is large enough, optimal localization may vary spatially, even within one model level. For instance, correlation structures of conventional variables are known to vary between precipitating and nonprecipitating zones (Montmerle and Berre, 2010), so that localization may be thought to vary accordingly.

Rapid implementations of the last two schemes are likely to have prohibitive numerical costs. Furthermore, variable-dependent scheme should give a specific attention to localization of crosscovariances between variables from two different sets.

An alternative solution would consist in using a kind of adaptive localization. Among existing schemes, an interesting possibility would be the use of a scale-dependent localization scheme (SDL, Buehner and Shlyaeva, 2015), that has been recently implemented in the experimental 3D-EnVar of AROME-France (Caron et al., 2018). Indeed, optimal localization lengths might be correlated with the spatial scale of error covariance structures, both horizontally and vertically. Hence, a scale-dependent localization would indirectly be a kind of variable-dependent, day-dependent and level-dependent localization, as shown by Caron and Buehner, 2018. Yet, SDL schemes would remain computationally expensive compared to single-length localization schemes, the cost being multiplied by the number of spectral bands chosen in the algorithm. With three bands, the cost of SDL is likely to be similar to the cost of a variable-dependent localization scheme with three sets of variables. Besides, we are not aware of an SDL scheme that could simultaneously filter horizontal and vertical scales, though this perspective is mentioned by Caron and Buehner, 2018. 


\section{6 | CONCLUSION AND PERSPECTIVES}

The method of Ménétrier et al. (2015a) diagnoses localization lengths for ensemble-based covariances, based on the ensemble of forecast only. This method has been extended to positive and highly heterogeneous variables such as hydrometeors. To deal with the problem of zero-variance grid points, we have recourse to geographical masks based on sample variances. The effect of these masks is twofold. First, model levels and separations where all covariances are zero (and thus where no localization is needed) are directly rejected from the diagnosis. Then, the step of spatial sampling related to the ergodicity assumption is made more efficient by sampling directly among the non-zero variance pairs of each separation class. Both modifications make the diagnoses more robust. A simple quality criterion for the diagnoses is also implemented, based on the agreement of raw diagnosed localization and its fit by an analytical localization function.

Horizontal localization lengths in model space are then diagnosed for hydrometeor variables from eight ensembles describing forecast error for different weather situations in 2018. For 50-member ensembles, hydrometeor localization lengths are mainly diagnosed between $20-80 \mathrm{~km}$, with large values (around $100 \mathrm{~km}$ ) for cloud water and some extreme values on one day near the tropopause for ice crystals and snow. In line with previous studies, larger typical values (from $100 \mathrm{~km}$ to $300 \mathrm{~km}$ ) are found for conventional variables, with higher values in the stratosphere. Though localization profiles are highly dependent on vertical level and weather situation, a level-by-level and day-by-day ratio shows that horizontal localization lengths diagnosed for hydrometeors are constantly shorter by a factor 2 to 6 compared to conventional variables.

Vertical localization lengths are also dependent on level and weather situation. Similar ranges of values are found for conventional variables, cloud water and ice crystals. Precipitating hydrometeors exhibit larger vertical localization structures and more abrupt transitions to zero values than other variables.

The absolute values of localization lengths given here can be extrapolated to different ensemble sizes: shorter lengths for smaller ensembles, larger ones for bigger ensembles. It should be noted though that the choice of ensemble size is not supposed to affect the main results of this study: day-to-day variability, dependence to vertical level, comparisons from variable to variable. Similarly, these results can reasonably be thought to extend to any convective scale model with a single-moment microphysics scheme.

Logarithm transforms or Gaussian anamorphosis are sometimes invoked as a workaround to deal with the non-Gaussian behavior of hydrometeors (Bocquet et al., 2010). The method used herein could be straightforwardly applied to such transformed ensembles, to see how our results extend to transformed hydrometeor fields.

Following the recommendations of this study about localization schemes for hydrometeor variables, cycling experiments are currently being performed at Météo-France to compare a single-localization, a variable-dependent localization and a scale-dependent localization schemes. The potential gain in skill will be weighed against the higher cost in computation power and time. In such assimilation experiments, hydrometeor increments can be produced even without hydrometeor observations, by 
projecting conventional increments to hydrometeor variables through cross-covariances of the $\mathbf{B}$ matrix. Hence, localization diagnoses for cross-covariances between hydrometeors and conventional variables would be worth studying in future research.

\section{7 | ACKNOWLEDGEMENTS}

The paper benefited from the careful reading of Loïk Berre. The PhD thesis of M. Destouches is funded by the French Ministère de l'Enseignement supérieur, de la Recherche et de l'Innovation, through a grant for alumni from the École polytechnique. The LEFE program (Les Enveloppes Fluides et l'Environnement) of the INSU-CNRS (Institut National des Sciences de l'Univers - Centre National de la Recherche Scientifique) funded part of this research within the framework of the Hydre project. The BUMP package (B matrix on an Unstructured Mesh Package) with all developments mentioned here and more is available on the SABER (StAtic Background Error Representation) github repository of the Joint Center for Satellite Data Assimilation: https://github.com/jcsda/saber. As of today, this repository is still private. However, it will become public with the first release of the JEDI (Joint Effort for Data assimilation Integration) system, scheduled for the fall of 2020.

\section{A I COMPUTING THE ANALYTICAL LOCALIZATION FUNCTION}

A few precisions are given about the computation of the analytical localization from $r_{h}, r_{v}$ and $\alpha$ profiles. For the sake of clarity, we first omit the normalization factors $\alpha(z)$.

For a given separation class $\left(z, d_{z}, d_{h}\right)$, how is the analytical localization $L^{\text {ana }}\left(z, d_{z}, d_{h}\right)$ computed? An intuitive choice would be:

$$
L^{\mathrm{ana}}\left(z, d_{z}, d_{h}\right)=\exp \left(-\frac{\Delta\left(z, d_{z}, d_{h}\right)^{2}}{2}\right)
$$

where $\left(\Delta\left(z, d_{z}, d_{h}\right)\right.$ is the normalized distance associated to the separation class:

$$
\Delta\left(z, d_{z}, d_{h}\right)=\sqrt{\left(\frac{d_{h}}{r_{h}(z)}\right)^{2}+\left(\frac{d_{z}}{r_{v}(z)}\right)^{2}} .
$$

The associated approximation using the correlation function from Gaspari and Cohn (1999) would be $L^{\text {ana }}\left(z, d_{z}, d_{h}\right)=\operatorname{GC99}\left(\Delta\left(z, d_{z}, d_{h}\right)\right)$, where GC99 denotes the approximation of a Gaussian with a unit Daley length-scale. The problem with this formulation comes from its asymmetry: why choosing $r_{v}(z)$ and not $r_{v}\left(z+d_{z}\right)$ to normalize vertical separation? $L^{\text {ana }}\left(z, d_{z}, d_{h}\right)$ and $L^{\text {ana }}\left(z+d_{z},-d_{z}, d_{h}\right)$ would differ here, making the underlying localization matrix non-symmetric: localization between levels $z$ and $z+d_{z}$ would be different from localization between levels $z+d_{z}$ and $z$.

Another candidate ensuring symmetry of the localization function shall be found for $\Delta$. Plus, we require that the distance between points at different levels $z_{i}$ and $z_{j}, i \leq j$, accounts for all intermediate 
values $r_{h}\left(z_{k}\right)$ and $r_{v}\left(z_{k}\right), i \leq k \leq j$, and not only for extreme values at levels $z_{i}$ and $z_{j}$.

To do so, at a given level $z$, we associate to each possible values of $d_{h}$ and $d_{z}$ a point on the 2D plane of possible horizontal and vertical separations. This plane is discretized has explained in section 2.2 .

We first define a locally normalized distance $\delta$ between two neighboring points $\left(d_{1}^{h}, d_{1}^{z}\right)$ and $\left(d_{2}^{h}, d_{2}^{z}\right)$, i.e. points that are horizontally, vertically or diagonally neighbors on the discrete $2 \mathrm{D}$ plane. We naturally have:

$$
\delta\left(\left(d_{1}^{h}, d_{1}^{z}\right),\left(d_{2}^{h}, d_{2}^{z}\right)\right)=\sqrt{\left(\frac{\left|d_{1}^{h}-d_{2}^{h}\right|}{r_{h}\left(z_{12}\right)}\right)^{2}+\left(\frac{\left|d_{1}^{z}-d_{2}^{z}\right|}{r_{v}\left(z_{12}\right)}\right)^{2}}
$$

where $r_{h}\left(z_{12}\right)$ is the mean of $r_{h}$ at levels $z+d_{1}^{z}$ and $z+d_{2}^{z}$, and similarly for $r_{v}\left(z_{12}\right)$.

Then, we define as path any sequence of points $\left(\left(d_{i}^{h}, d_{i}^{z}\right)\right)_{0 \leq i \leq p}$ such that two consecutive points are neighbors on the grid. The length of a path is naturally defined as the sum of locally normalized distances: $\sum_{i=0}^{p-1} \delta\left(\left(d_{h}^{i}, d_{z}^{i}\right),\left(d_{h}^{i+1}, d_{z}^{i+1}\right)\right)$.

We now choose as normalized distance $\Delta\left(z, d_{z}, d_{h}\right)$ for separation class $\left(z, d_{z}, d_{h}\right)$ the length of the shortest path from zero separation to $\left(d_{h}, d_{z}\right)$, "shortest" meaning "of minimal length".

Finally, the obtained localization function is normalized by the factor $\alpha(z)$, still taking care of preserving the symmetry property: $L^{\text {ana }}\left(z_{i}, z_{j}, d_{h}\right) \leftarrow L^{\text {ana }}\left(z_{i}, z_{j}, d_{h}\right) \times \sqrt{\alpha\left(z_{i}\right) \alpha\left(z_{j}\right)}$.

\section{B I FITTING THE ANALYTICAL LOCALIZATION FUNCTION}

In practice, a simple implementation of the fit process leads to $r_{h}$ and $r_{v}$ profiles that exhibit sharp, high-frequency features. The non reproducibility from one run to another suggest that these 2-level frequency features are numerical noise, resulting from an over-fitting of the raw localization function. To prevent this behavior, to make the diagnoses more robust and to remain close to smooth profiles and Gaussian shapes of the analytical function, two elements are added to the fit step.

1. Penalty terms are included in the cost function, penalizing high-curvature profiles. The penalty terms are sums of discrete normalized second order derivatives along the vertical direction, and are zero if the profiles are linear.

2. The control variables $r_{h}, r_{v}$ and $\alpha$ of the minimization are shortened by controlling the profiles only one level out of two. The missing intermediate levels are found by linear interpolation before computation of $L^{\text {ana }}$.

Both modifications are necessary in practice. The cost function being minimized to obtain $r_{h}$ and $r_{v}$ is 


$$
\begin{array}{r}
J\left(r_{h}^{\prime}, r_{v}^{\prime}, \alpha^{\prime}\right)=\left\|L^{\text {ana }}\left(r_{h}, r_{v}, \alpha\right)-L^{r a w}\right\|_{2}^{2} \\
+J^{\text {curv }}\left(r_{h}\right)+J^{\text {curv }}\left(r_{v}\right)+J^{\text {curv }}(\alpha)
\end{array}
$$

where the norm is computed over all values of $z, d_{z}$ and $d_{h}$. This approach allows to extract localization length profiles while exploiting the full information of the raw localization function, which is more robust than deducing them from raw localization at zero horizontal or vertical separation. The $r_{h}$ profile is linearly interpolated from the twice shorter $r_{h}^{\prime}, r_{v}$ is interpolated from $r_{v}^{\prime}$ and $\alpha$ from $\alpha^{\prime}$. If an excessive weight is given to the penalty terms $J^{\text {curv }}$, fit profiles tend towards linear profiles. Obtaining peaked profiles in section 5 shows that the penalty terms are correctly balanced relative to the first term. Plus, sharper localization profiles would be useless compared to the current difficulties in applying localization schemes with varying lengths (vertically constant localization lengths were used in Montmerle et al., 2018 for instance).

\section{I ACCEPTANCE TESTS FOR PROFILES OF LOCALIZATION LENGTHS}

As explained in section 3.4, for a given level $z, r_{h}(z)$ and $r_{v}(z)$ are simultaneously accepted or rejected, based on the confidence we have on the fit step.

1. The agreement between raw localization and analytical localization is assessed by computing a signal-to-noise ratio (SNR). Since the fit process is important only in the decaying zone of the function, the SNR is computed only "near the zero separation class" (within a one bin distance of the zero separation class, in the $d_{h}, d_{z}$ plane) and where the fit localization is above half its maximum value. Noise is estimated by the root mean square difference of raw and analytical localization functions, and signal is estimated by the mean value of raw localization in the decaying zone. Levels where the SNR is lower than 1 are rejected.

2. Insufficient hydrometeor contents are declared when there are not enough diagnosed points in the raw localization function at level $z$ to correctly fit the analytical function. Separation classes "near the zero separation class" (cf. point 1) should all have a valid localization value, diagnosed from at least 1000 pairs. Otherwise, $r_{h}(z)$ and $r_{v}(z)$ are rejected.

\section{REFERENCES}

Aksoy, Altuğ, David C Dowell, and Chris Snyder (2009). "A multicase comparative assessment of the ensemble Kalman filter for assimilation of radar observations. Part I: Storm-scale analyses”. In: Monthly Weather Review 137.6, pp. 18051824. DOI: $10.1175 / 2008 \mathrm{mwr} 2691.1$.

Anderson, Jeffrey L. (2012). "Localization and Sampling Error Correction in Ensemble Kalman Filter Data Assimilation”. In: Monthly Weather Review 140.7, pp. 2359-2371. ISSN: 0027-0644. DOI: 10.1175/mwr-d-11-00013.1. 
Auligné, Thomas, Andrew Lorenc, Yann Michel, Thibaut Montmerle, A Jones, M Hu, and Jimy Dudhia (2011). “Toward a new cloud analysis and prediction system". In: Bulletin of the American Meteorological Society 92.2, pp. 207-210. DOI: $10.1175 / 2010$ bams 2978.1.

Bannister, Ross N (2008). "A review of forecast error covariance statistics in atmospheric variational data assimilation. I: Characteristics and measurements of forecast error covariances". In: Quarterly Journal of the Royal Meteorological Society 134.637, pp. 1951-1970. DOI: 10.1002/qj.339.

Bannister, Ross Noel, Hristo Chipilski, and Oscar Martinez-Alvarado (2019). "Techniques and challenges in the assimilation of atmospheric water observations for numerical weather prediction towards convective scales". In: Quarterly Journal of the Royal Meteorological Society. DOI: 10.1002/qj.3652.

Berre, Loïk and Gérald Desroziers (2010). "Filtering of Background Error Variances and Correlations by Local Spatial Averaging: A Review". In: Monthly Weather Review 138.10, pp. 3693-3720. DOI: 10.1175/2010mwr3111.1.

Berre, Loïk, Hubert Varella, and Gérald Desroziers (2015). "Modelling of flow-dependent ensemble-based backgrounderror correlations using a wavelet formulation in 4D-Var at Météo-France". In: Quarterly Journal of the Royal Meteorological Society 141.692, pp. 2803-2812. DOI: 10.1002/qj. 2565.

Bocquet, Marc, Carlos A Pires, and Lin Wu (2010). "Beyond Gaussian statistical modeling in geophysical data assimilation”. In: Monthly Weather Review 138.8, pp. 2997-3023. DOI: 10.1175/2010mwr3164.1.

Bouttier, François, Benoît Vié, Olivier Nuissier, and Laure Raynaud (2012). "Impact of stochastic physics in a convectionpermitting ensemble”. In: Monthly Weather Review 140.11, pp. 3706-3721. DOI: 10.1175/mwr-d-12-00031.1.

Brousseau, Pierre, Loïk Berre, François Bouttier, and Gérald Desroziers (2012). "Flow-dependent background-error covariances for a convective-scale data assimilation system". In: Quarterly Journal of the Royal Meteorological Society 138.663, pp. 310-322. DOI: 10.1002/qj.920.

Brousseau, Pierre, Yann Seity, Didier Ricard, and Julien Léger (2016). "Improvement of the forecast of convective activity from the AROME-France system". In: Quarterly Journal of the Royal Meteorological Society 142.699, pp. 22312243. DOI: $10.1002 / q j .2822$.

Buehner, Mark (2005). "Ensemble-derived stationary and flow-dependent background-error covariances: Evaluation in a quasi-operational NWP setting”. In: Quarterly Journal of the Royal Meteorological Society 131.607, pp. 1013-1043. DOI: $10.1256 / \mathrm{qj} \cdot 04.15$.

Buehner, Mark and Anna Shlyaeva (2015). "Scale-dependent background-error covariance localisation". In: Tellus A: Dynamic Meteorology and Oceanography 67.1, p. 28027. DOI: 10.3402/tellusa.v67.28027.

Caron, Jean-François and Mark Buehner (2018). "Scale-Dependent Background-Error Covariance Localization: Evaluation in a Global Deterministic Weather Forecasting System". In: Monthly Weather Review 146.5, pp. 1367-1381. DOI: 10.1175/mwr-d-17-0369.1.

Caron, Jean-François, Yann Michel, Thibaut Montmerle, and étienne Arbogast (2018). "Improving Background-Error Covariances in a 3D Ensemble-Variational Data Assimilation System for Regional NWP." In: Monthly Weather Review. DOI: $10.1175 / \mathrm{mwr}-\mathrm{d}-18-0248.1$.

Daley, Roger (1993). Atmospheric data analysis. 2. Cambridge university press.

Dowell, David C., Fuqing Zhang, Louis J. Wicker, Chris Snyder, and N. Andrew Crook (2004). "Wind and Temperature Retrievals in the 17 May 1981 Arcadia, Oklahoma, Supercell: Ensemble Kalman Filter Experiments". In: Monthly Weather Review 132.8, pp. 1982-2005. DOI: 10.1175/1520-0493 (2004) 132<1982: watrit>2.0. co; 2.

Dowell, David C, Louis J Wicker, and Chris Snyder (2011). "Ensemble Kalman filter assimilation of radar observations of the 8 May 2003 Oklahoma City supercell: Influences of reflectivity observations on storm-scale analyses". In: Monthly Weather Review 139.1, pp. 272-294. DOI: 10.1175/2010mwr3438.1.

Duda, Jeffrey D., Xuguang Wang, Yongming Wang, and Jacob R. Carley (2019). "Comparing the Assimilation of Radar Reflectivity Using the Direct GSI based Ensemble-Variational (EnVar) and Indirect Cloud Analysis Methods in Convection-Allowing Forecasts over the Continental US". In: Monthly Weather Review. DOI: 10 . 1175/mwr - d18-0171.1. 
Errico, Ronald M, Peter Bauer, and Jean-François Mahfouf (2007). "Issues regarding the assimilation of cloud and precipitation data". In: Journal of the Atmospheric Sciences 64.11, pp. 3785-3798. DOI: 10.1175/2006jas2044. 1.

Flowerdew, Jonathan (2015). "Towards a theory of optimal localisation”. In: Tellus A: Dynamic Meteorology and Oceanography 67.1, p. 25257. DOI: 10.3402/tellusa.v67.25257.

Gaspari, Gregory and Stephen E Cohn (1999). "Construction of correlation functions in two and three dimensions". In: Quarterly Journal of the Royal Meteorological Society 125.554, pp. 723-757. DOI: 10.1002/qj .49712555417.

Gustafsson, Nils, Tijana Janjić, Christoph Schraff, Daniel Leuenberger, Martin Weissmann, Hendrik Reich, Pierre Brousseau, Thibaut Montmerle, Eric Wattrelot, Antonín Bučánek, Máté Mile, Rafiq Hamdi, Magnus Lindskog, Jan Barkmeijer, Mats Dahlbom, Bruce Macpherson, Sue Ballard, Gordon Inverarity, Jacob Carley, Curtis Alexander, David Dowell, Shun Liu, Yasutaka Ikuta, and Tadashi Fujita (2018). "Survey of data assimilation methods for convectivescale numerical weather prediction at operational centres". In: Quarterly Journal of the Royal Meteorological Society 144.713, pp. 1218-1256. DOI: 10.1002/qj.3179.

Hamill, Thomas M, Jeffrey S Whitaker, and Chris Snyder (2001). "Distance-dependent filtering of background error covariance estimates in an ensemble Kalman filter". In: Monthly Weather Review 129.11, pp. 2776-2790. DOI: 10 . 1175/1520-0493 (2001) 129<2776: ddfobe>2.0.co;2.

Houtekamer, Peter L and Herschel L Mitchell (2001). "A sequential ensemble Kalman filter for atmospheric data assimilation”. In: Monthly Weather Review 129.1, pp. 123-137. DOI: 10.1175/1520-0493 (2001) 129<0123: asekff>2.0. co;2.

Houtekamer, Peter L and Fuqing Zhang (2016). "Review of the ensemble Kalman filter for atmospheric data assimilation". In: Monthly Weather Review 144.12, pp. 4489-4532. DOI: 10.1175/mwr-d-15-0440.1.

Johnson, Aaron, Xuguang Wang, Jacob R. Carley, Louis J. Wicker, and Christopher Karstens (2015). "A Comparison of Multiscale GSI-Based EnKF and 3DVar Data Assimilation Using Radar and Conventional Observations for Midlatitude Convective-Scale Precipitation Forecasts". In: Monthly Weather Review 143.8, pp. 3087-3108. DOI: 10.1175/ mwr-d-14-00345.1.

Kong, Rong, Ming Xue, and Chengsi Liu (2018). "Development of a Hybrid En3DVar Data Assimilation System and Comparisons with 3DVar and EnKF for Radar Data Assimilation with Observing System Simulation Experiments". In: Monthly Weather Review 146.1, pp. 175-198. DOI: 10.1175/mwr-d-17-0164.1.

Lascaux, Franck, Evelyne Richard, and Jean-Pierre Pinty (2006). "Numerical simulations of three different MAP IOPs and the associated microphysical processes". In: Quarterly Journal of the Royal Meteorological Society 132.619, pp. 1907-1926. DOI: 10.1256/qj.05.197.

Lei, Lili, Jeffrey L. Anderson, and Glen S. Romine (2015). "Empirical Localization Functions for Ensemble Kalman Filter Data Assimilation in Regions with and without Precipitation”. In: Monthly Weather Review 143.9, pp. 3664-3679. ISSN: 0027-0644. DOI: 10.1175/mwr-d-14-00415.1.

Lorenc, Andrew C. (2017). "Improving ensemble covariances in hybrid variational data assimilation without increasing ensemble size”. In: Quarterly Journal of the Royal Meteorological Society 143.703, pp. 1062-1072. ISSN: 0035-9009. DOI: $10.1002 / q j .2990$.

Ménétrier, Benjamin, Thibaut Montmerle, Loïk Berre, and Yann Michel (2014). "Estimation and diagnosis of heterogeneous flow-dependent background-error covariances at the convective scale using either large or small ensembles". In: Quarterly Journal of the Royal Meteorological Society 140.683, pp. 2050-2061. DOI: 10.1002/qj .2267.

Ménétrier, Benjamin, Thibaut Montmerle, Yann Michel, and Loïk Berre (2015a). "Linear Filtering of Sample Covariances for Ensemble-Based Data Assimilation. Part I: Optimality Criteria and Application to Variance Filtering and Covariance Localization”. In: Monthly Weather Review 143.5, pp. 1622-1643. DOI: 10.1175/mwr-d-14-00157.1.

- (2015b). "Linear filtering of sample covariances for ensemble-based data assimilation. Part II: Application to a convective-scale NWP model". In: Monthly Weather Review 143.5, pp. 1644-1664. DOI: 10 . 1175 /mwr - d - 14 00156.1 . 
Meng, Deming, Yaodeng Chen, Hongli Wang, Yufang Gao, Roland Potthast, and Yuanbing Wang (2019). "The evaluation of EnVar method including hydrometeors analysis variables for assimilating cloud liquid/ice water path on prediction of rainfall events". In: Atmospheric Research 219, pp. 1-12. DOI: 10.1016/j . atmosres .2018.12.017.

Michel, Yann, Thomas Auligné, and Thibaut Montmerle (2011). "Heterogeneous convective-scale background error covariances with the inclusion of hydrometeor variables". In: Monthly Weather Review 139.9, pp. 2994-3015. DOI: 10.1175/2011mwr3632. 1.

Michel, Yann, Benjamin Ménétrier, and Thibaut Montmerle (2016). “Objective filtering of the local correlation tensor”. In: Quarterly Journal of the Royal Meteorological Society 142.699, pp. 2314-2323. DOI: 10.1002/qj .2824.

Montmerle, Thibaut and Loïk Berre (2010). "Diagnosis and formulation of heterogeneous background-error covariances at the mesoscale". In: Quarterly Journal of the Royal Meteorological Society 136.651, pp. 1408-1420. DOI: 10.1002/ qj. 655 .

Montmerle, Thibaut, Yann Michel, Etienne Arbogast, Benjamin Ménétrier, and Pierre Brousseau (2018). "A 3D ensemble variational data assimilation scheme for the limited-area AROME model: Formulation and preliminary results". In: Quarterly Journal of the Royal Meteorological Society 144.716, pp. 2196-2215. DOI: 10.1002/qj .3334.

Necker, Tobias, Stefan Geiss, Martin Weissmann, Juan Ruiz, Takemasa Miyoshi, and Guo-Yuan Lien (2020). “A convective-scale 1000-member ensemble simulation and potential applications". In: Quarterly Journal of the Royal Meteorological Society. DOI: 10.1002/qj.3744.

Palmer, Tim N., Roberto Buizza, Francisco J Doblas-Reyes, Thomas Jung, Martin Leutbecher, Glenn J. Shutts, Martin Steinheimer, and Antje Weisheimer (2009). Stochastic parametrization and model uncertainty. Tech. rep. 598. ECMWF. DOI: 10.21957/PS8GBWBDV.

Perianez, Africa, Hendrik Reich, and Roland Potthast (2014). "Optimal localization for ensemble Kalman filter systems". In: Journal of the Meteorological Society of Japan. Ser. II 92.6, pp. 585-597. DOI: 10.2151/jms j.2014-605.

Seity, Yann, Pierre Brousseau, Sophie Malardel, Gwenaëlle Hello, Pierre Bénard, François Bouttier, Christine Lac, and Valérie Masson (2011). "The AROME-France convective-scale operational model”. In: Monthly Weather Review 139.3, pp. 976-991. DOI: 10.1175/2010mwr3425.1.

Snyder, Chris and Fuqing Zhang (2003). "Assimilation of simulated Doppler radar observations with an ensemble Kalman filter.” In: Monthly Weather Review 131.8. DOI: 10.1175//2555.1.

Sobash, Ryan A. and David J. Stensrud (2013). "The Impact of Covariance Localization for Radar Data on EnKF Analyses of a Developing MCS: Observing System Simulation Experiments”. In: Monthly Weather Review 141.11, pp. 36913709. DOI: $10.1175 / \mathrm{mwr}-\mathrm{d}-12-00203.1$.

Zhang, Sara Q, Milija Zupanski, Arthur Y Hou, Xin Lin, and Samson H Cheung (2013). "Assimilation of precipitationaffected radiances in a cloud-resolving WRF ensemble data assimilation system". In: Monthly Weather Review 141.2, pp. 754-772. DOI: $10.1175 / \mathrm{mwr}-\mathrm{d}-12-00055.1$. 